Working Papers

Institute of

Mathematical

Economics

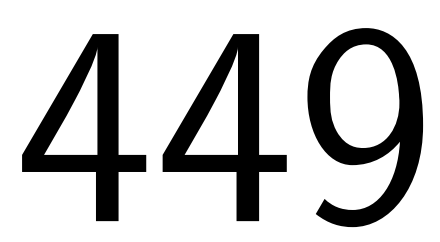

June 2011

\title{
Competitive Outcomes and the Inner Core of NTU Market Games
}

Sonja Brangewitz and Jan-Philip Gamp
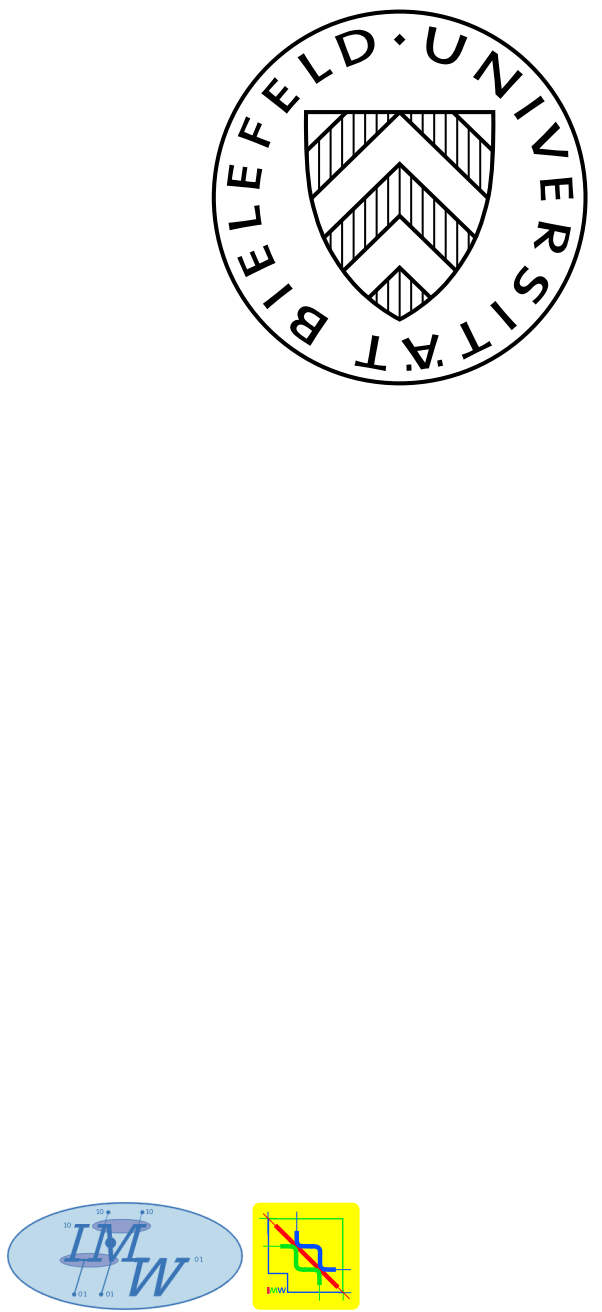


\title{
Competitive Outcomes and the Inner Core of NTU Market Games *
}

\author{
Sonja Brangewitz and Jan-Philip Gamp ${ }^{\dagger}$
}

June 1, 2011

\begin{abstract}
We consider the inner core as a solution concept for cooperative games with nontransferable utility (NTU) and its relationship to competitive equilibria of markets that are induced by an NTU game. We investigate the relationship between certain subsets of the inner core for NTU market games and competitive payoff vectors of markets linked to the NTU market game. This can be considered as the case in between the two extreme cases of Qin (1993). We extend the results of Qin (1993) to a large class of closed subsets of the inner core: Given an NTU market game we construct a market depending on a given closed subset of its inner core. This market represents the game and further has the given set as the set of payoffs of competitive equilibria. It turns out that this market is not determined uniquely and thus we obtain a class of markets with the desired property.
\end{abstract}

Keywords and Phrases: Market Games, Competitive Payoffs, Inner Core JEL Classification Numbers: C71, D51

\footnotetext{
${ }^{*}$ We are grateful for discussions with and comments from Jean-Marc Bonnisseau and Walter Trockel. Financial Support through the International Research Training Group EBIM, "Economic Behavior and Interaction Models", the German Academic Exchange Service (DAAD) and the Franco-German University (DFH - UFA) is gratefully acknowledged.

$\dagger$ Institute of Mathematical Economics, Bielefeld University, P.O. Box 100131, 33501 Bielefeld, Germany and Centre d'Economie de la Sorbonne, Université Paris 1 Panthéon Sorbonne, 106-112 Boulevard de l'Hôpital, 75647 Paris Cedex 13, France; sonja.brangewitz@wiwi.uni-bielefeld.de and janphilip.gamp@wiwi.uni-bielefeld.de
} 


\section{Introduction}

The idea to consider cooperative games as economies or markets goes back to Shapley and Shubik (1969). They look at TU market games. These are cooperative games with transferable utility (TU) that are in a certain sense linked to economies or markets. More precisely, a market is said to represent a game if the set of utility allocations a coalition can reach in the market coincides with the set of utility allocations a coalition obtains according to the coalitional function of the game. If there exists a market that represents a game, then this game is called a market game. Shapley and Shubik (1969) prove the identity of the class of totally balanced TU games with the class of TU market games. Furthermore, Shapley and Shubik (1975) show that starting with a TU market game every payoff vector in the core of that game is competitive in a certain market, called direct market, and that for any given point in the core there exists at least one market that has this payoff vector as its unique competitive payoff vector.

Cooperative games with non-transferable utility (NTU) can be considered as a generalization of TU games, where the transfer of the utility within a coalition does not take place at a fixed rate. In this paper we consider NTU market games. After Shapley and Shubik (1969), Billera and Bixby (1974) investigated the NTU case and obtained similar results for compactly convexly generated NTU games. Analogously to the result of Shapley and Shubik (1969) they show that every totally balanced NTU game, that is compactly convexly generated, is a market game. The inner core is a refinement of the core for NTU games. A point is in the inner core if there exists a transfer rate vector, such that - given this transfer rate vector - no coalition can improve even if utility can be transferred within a coalition according to this vector. So, an inner core point is in the core of an associated hyperplane game where the utility can be transferred according to the transfer rate vector. Qin (1993) shows, verifying a conjecture of Shapley and Shubik (1975), that the inner core of a market game coincides with the set of competitive payoff vectors of the induced market of that game. Moreover, he shows that for every NTU market game and for any given point in its inner core there exists a market that represents the game and further has this given inner core point as its unique competitive payoff vector.

Similarly to the approach of Billera and Bixby (1974), Inoue (2010b) uses coalition production economies as in Sun et al. (2008) instead of markets. Inoue (2010b) shows that every compactly generated NTU game can be represented by a coalition production economy. Moreover, he proves that there exists a coalition production economy whose set of competitive payoff vectors coincides with the inner core of the balanced cover of the original NTU game.

Here we consider the classical approach using markets. We investigate the case in 
between the two extreme cases of Qin (1993), where on the one hand there exists a market that has the complete inner core as its set of competitive payoff vectors and on the other hand there is a market that has a given inner core point as its unique competitive payoff vector. We extend the results of Qin (1993) to closed subsets of the inner core: Given an NTU market game we construct a market depending on a given closed subset of the inner core. This market represents the game and further has the given set as the set of payoffs of competitive equilibria. It turns out that this market is not determined uniquely. Several parameters in our construction can be chosen in different ways. Thus, we obtain a class of markets with the desired property.

Shapley and Shubik (1975) remark that in the TU case their result can be extended to any closed and convex subset of the core. Whether a similar result analogously to the one of Shapley and Shubik (1975) holds for NTU market games, was up to now not clear. Our result shows, that in the NTU case it is even possible to focus on closed, typically non-convex, subsets of the inner core.

The inner is one solution concept for NTU games. Extending the results of Qin (1993) to closed subsets of the inner core means in particular to show such a result for all solution concepts selecting closed subsets of the inner core.

\section{$2 \quad$ NTU market games}

Let $N=\{1, \ldots, n\}$ with $n \in \mathbb{N}$ and $n \geq 2$ be a set of players. Let $\mathcal{N}=\{S \subseteq N \mid S \neq \emptyset\}$ be the set of coalitions. Define for a coalition $S \in \mathcal{N}$ the following sets $\mathbb{R}^{S}=\left\{x \in \mathbb{R}^{n} \mid x_{i}=\right.$ 0 if $i \notin S\} \subseteq \mathbb{R}^{n}, \mathbb{R}_{+}^{S}=\left\{x \in \mathbb{R}^{S} \mid x_{i} \geq 0\right.$ for all $\left.i \in S\right\} \subseteq \mathbb{R}_{+}^{n}, \mathbb{R}_{++}^{S}=\left\{x \in \mathbb{R}^{S} \mid x_{i}>\right.$ 0 for all $i \in S\} \subseteq \mathbb{R}_{++}^{n}$. For a vector $a \in \mathbb{R}^{n}$ and a coalition $S \in \mathcal{N}$ let $a^{S}$ denote the vector, where for $i \in S$ we have $a_{i}^{S}=a_{i}$ and $a_{j}^{S}=0$ for $j \notin S$. Moreover, for $a \in \mathbb{R}^{n}$ and $b \in \mathbb{R}^{n}$ denote the inner product by $a \cdot b=\sum_{i=1}^{n} a_{i} b_{i}$ and the Hadamard product by $a \circ b=\left(a_{1} b_{1}, \ldots, a_{n} b_{n}\right)$.

An $N T U$ (non-transferable utility) game is a pair $(N, V)$, that consists of a player set $N=\{1, \ldots, n\}$ and a coalitional function $V$, which defines for every coalition the utility allocations this coalition can reach, regardless of what the other players outside this coalition do. Hence, define the coalitional function $V$ from the set of coalitions, $\mathcal{N}$, to the set of non-empty subsets of $\mathbb{R}^{n}$, such that for every coalition $S \in \mathcal{N}$ we have $V(S) \subseteq \mathbb{R}^{S}$, $V(S)$ is non-empty and $V(S)$ is $S$-comprehensive, meaning $V(S) \supseteq V(S)-\mathbb{R}_{+}^{S}$.

The literature on NTU market games, as for example Billera and Bixby (1974) and Qin (1993), considers NTU games that are compactly and convexly generated. An NTU game $(N, V)$ is compactly (convexly) generated if for all coalitions $S \in \mathcal{N}$ there exists a compact (convex) set $C^{S} \subseteq \mathbb{R}^{S}$ such that the coalitional function has the form $V(S)=C^{S}-\mathbb{R}_{+}^{S}$. 
Given a player set $N=\{1, \ldots, n\}$ the set of balancing weights is defined by $\Gamma\left(e^{N}\right)=$ $\left\{\left(\gamma_{S}\right)_{S \subseteq N} \mid \gamma_{S} \geq 0 \forall S \subseteq N, \sum_{S \subseteq N} \gamma_{S} e^{S}=e^{N}\right\}$. The balancing weights can be interpreted in the following way: Every player $i$ has one unit of time that he can split over all the coalitions, he is a member of, with the constraint that a coalition has to agree on a common weight. Thereby, each player has to spend all his time. The weight $\gamma_{S}$ can be seen as well as the intensity with which each player participates in the coalition $S \in \mathcal{N}$. In particular, if we have a partition of the player set into a coalition $S$ and its complement $N \backslash S$ a balancing weight can be defined by $\gamma_{S}=\gamma_{N \backslash S}=1$ and $\gamma_{T}=0$ for all other coalitions $T$ except for $S$ and $N \backslash S$. An NTU game $(N, V)$ is balanced if for all balancing weights $\gamma \in \Gamma\left(e^{N}\right)$ we have $\sum_{S \subseteq N} \gamma_{S} V(S) \subseteq V(N)$. Moreover, an NTU game $(N, V)$ is totally balanced if it is balanced in all subgames. This means for all coalitions $T \in \mathcal{N}$ and for all balancing weights $\gamma \in \Gamma\left(e^{T}\right)=\left\{\left(\gamma_{S}\right)_{S \subseteq T} \mid \gamma_{S} \geq 0 \forall S \subseteq T, \sum_{S \subseteq T} \gamma_{S} e^{S}=e^{T}\right\}$ we have $\sum_{S \subseteq T} \gamma_{S} V(S) \subseteq V(T)$.

In order to define an NTU market game we first consider the notion of a market which is less general than the notion of an economy according to for example Arrow and Debreu (1954). In a market the number of consumers coincides with the number of producers. Each consumer has his own private production set. In contrast to the usual notion of an economy a market is assumed to have concave and not just quasi concave utility functions.

Definition (market). A market is given by $\mathcal{E}=\left\{\left(X^{i}, Y^{i}, \omega^{i}, u^{i}\right)_{i \in N}\right\}$ where for every individual $i \in N$

- $X^{i} \subseteq \mathbb{R}_{+}^{\ell}$ is a non-empty, closed and convex set, the consumption set, where $\ell \geq 1$, $\ell \in \mathbb{N}$ is the number of commodities,

- $Y^{i} \subseteq \mathbb{R}^{\ell}$ is a non-empty, closed and convex set, the production set, such that $Y^{i} \cap \mathbb{R}_{+}^{\ell}=\{0\}$

- $\omega^{i} \in X^{i}-Y^{i}$, the initial endowment vector,

- and $u^{i}: X^{i} \rightarrow \mathbb{R}$ is a continuous and concave function, the utility function.

As pointed out before in a market each consumer is assumed have his own private production set. This assumption is not as restrictive as it appears to be. A given private ownership economy can be transformed into an economy with the same number of consumers and producers without changing the set of competitive equilibria or possible utility allocations, see for example Qin and Shubik (2009, section 4).

In the following, we often consider markets where $X^{i} \subseteq \mathbb{R}_{+}^{k n}$ with $k, n \in \mathbb{N}$. Then, consumption vectors are usually written as $x^{i}=\left(x^{(1) i}, \ldots, x^{(k) i}\right) \in X^{i}$ where $x^{(m) i} \in \mathbb{R}_{+}^{n}$ 
for $m=1, \ldots, k$. In a sense, we divide the $k n$ consumption goods in $k$ consecutive groups of $n$ goods. The vector $x^{(m) i}$ is the $m^{\text {th }}$ group of $n$ consumption goods of the consumption vector $x^{i}$. We use an analogous notation for the production goods and price vectors.

Given a market we define which allocations are considered as feasible for some coalition $S \in \mathcal{N}$. An $S$-allocation is a tuple $\left(x^{i}\right)_{i \in S}$ such that $x^{i} \in X^{i}$ for each $i \in S$. The set of feasible $S$-allocations is given by

$$
F(S)=\left\{\left(x^{i}\right)_{i \in S} \mid x^{i} \in X^{i} \text { for all } i \in S, \sum_{i \in S}\left(x^{i}-\omega^{i}\right) \in \sum_{i \in S} Y^{i}\right\} .
$$

Hence, an $S$-allocation is feasible if there exist for all $i \in S$ production plans $y^{i} \in Y^{i}$ such that $\sum_{i \in S}\left(x^{i}-\omega^{i}\right)=\sum_{i \in S} y^{i}$. We refer to a feasible $S$-allocation in the following together with suitable production plans as a feasible $S$-allocation $\left(x^{i}\right)_{i \in S}$ with $\left(y^{i}\right)_{i \in S}$.

In the definition of feasibility it is implicitly assumed that by forming a coalition the available production plans are the sum of the individually available production plans. This approach is different from the idea to use coalition production economies, where every coalition has already in the definition of the economy its own production possibility set. Nevertheless, a market can be transformed into a coalition production economy by defining the production possibility set of a coalition as the sum of the individual production possibility sets.

Given the notion of a market and of feasible allocations for coalitions $S \in \mathcal{N}$ we define an NTU market game in the following way:

Definition (NTU market game). An NTU game $(N, V)$ that is representable by a market is an $N T U$ market game. This means there exists a market $\mathcal{E}$ such that $\left(N, V_{\mathcal{E}}\right)=(N, V)$ with

$$
V_{\mathcal{E}}(S)=\left\{u \in \mathbb{R}^{S} \mid \exists\left(x^{i}\right)_{i \in S} \in F(S), u_{i} \leq u^{i}\left(x^{i}\right), \forall i \in S\right\} .
$$

For an NTU market game there exists a market such that the set of utility allocations a coalition can reach according to the coalitional function coincides with the set of utility allocations that are generated by feasible $S$-allocations in the market or that give less utility than some feasible $S$-allocation.

One of the main results on NTU market games in Billera and Bixby (1974) is the following:

Theorem (2.1, Billera and Bixby (1974)). An NTU game $(N, V)$ is an NTU market game if and only if it is totally balanced and compactly convexly generated.

Hence, in order to study NTU market games, it is sufficient to look at those NTU games that are totally balanced and compactly convexly generated. 
For the succeeding analysis, it will be useful to shift a given NTU game in the following way (compare Billera and Bixby (1973, Proposition 2.2)): Given a vector $c \in \mathbb{R}^{n}$ define the coalitional function $(V+c)$ via $(V+c)(S)=V(S)+\sum_{i \in S} c_{i}$. To represent a shifted game by a market we have to shift the utility function of agent $i$ by $c_{i}$. Hence, the shifted game with coalitional function $(V+c)$ is again a market game. Furthermore, shifting the utility functions of the agents does not change the set of competitive equilibria. Having this idea of shifting in mind we will focus in some proofs on games where for every coalition $S \in \mathcal{N}$ we have $C^{S} \subseteq \mathbb{R}_{++}^{S}$.

To prove the above result Billera and Bixby (1974) introduce the notion of an induced market that arises from a compactly convexly generated NTU game.

Definition (induced market). Let $(N, V)$ be a compactly convexly generated NTU game. The induced market of the game $(N, V)$ is defined by

$$
\mathcal{E}_{V}=\left\{\left(X^{i}, Y^{i}, u^{i}, \omega^{i}\right)_{i \in N}\right\}
$$

with for each individual $i \in N$

- the consumption set $X^{i}=\mathbb{R}_{+}^{n} \times\{0\} \subseteq \mathbb{R}^{2 n}$,

- the production set $Y^{i}=$ convexcone $\left[\bigcup_{S \in \mathcal{N}}\left(C^{S} \times\left\{-e^{S}\right\}\right)\right] \subseteq \mathbb{R}^{2 n}$,

- the initial endowment vector $\omega^{i}=\left(0, e^{\{i\}}\right)$,

- and the utility function $u^{i}: X^{i} \rightarrow \mathbb{R}$ with $u^{i}\left(x^{i}\right)=x_{i}^{(1) i}$.

It can easily be seen that this is a market according to the previous definition. Note that in an induced market we have input and output goods. Initially every consumer owns one unit of his personal input good that can only be used for the production process. By using his input good the consumer can get utility just from his personal output good. The consumption and production set are the same for every player. Just the utility functions and the initial endowments are dependent on the player.

The individual production sets in an induced market are convex cones and identical for all agents. In this situation taking the sum over production sets of some agents leads to the same production set. Setting $Y=\sum_{i \in N} Y^{i}$ the condition for feasibility of $S$ allocations reduces to $\sum_{i \in S}\left(x^{i}-\omega^{i}\right) \in Y$. Furthermore, for convex-cone technologies the competitive equilibrium profits are equal to 0 . This means that in equilibrium we do not have to specify shares of the production as it usually done in private ownership economies.

Thus, as long as the individual production sets are convex cones and identical for all 
agents, we could alternatively consider a model for the production where we have only one production set for all agents and possible coalitions without specifying the shares. This model could be used instead of the production setup in the definition of a market.

In the definition of the induced market it is assumed that every individual has already the production possibilities, that become available if coalitions form, included in his personal production set. This means he already knows everything that can be produced in the different coalitions, even if he does not possess the necessary input commodities himself. Starting with an NTU game the utility allocations a coalition can reach in the derived induced market are not described by defining production sets individually for every coalition but by using input and output commodities. A utility allocation, that is reachable in the NTU game by a coalition $S$, is reachable in the induced market by the same coalition if the individuals pool their initial endowments using "one general" production possibility set. Utility allocations that require the cooperation of individuals outside the coalition $S$ are technologically possible but can actually not be produced as the input commodities of these individuals are needed. In contrast to this interpretation in coalition production economies every coalition has its own production set.

The main proof of the above theorem from Billera and Bixby (1974) relies on Billera (1974). In a similar manner as Shapley and Shubik (1969), he starts with an NTU game, $(N, V)$, and looks at the induced market of that game, $\mathcal{E}_{V}$, and afterwards at the NTU game that is induced by the induced market, $V_{\mathcal{E}_{V}}$. He shows that this game coincides with the totally balanced cover of the game $(N, V)$.

The next step is to investigate the existing literature on and to study the relationship between solution concepts in cooperative game theory, as the inner core, and those in general equilibrium theory, as the notion of a competitive equilibrium. Analogously to the TU case of Shapley and Shubik (1975), Qin (1993) shows that the inner core of an NTU market game coincides with the set of competitive payoff vectors of the induced market of that game. Moreover, he shows that for every NTU market game and for any given point in its inner core, there is a market that represents the game and further has the given inner core point as its unique competitive payoff vector. Before we extend the results of Qin (1993) we recall the basic definitions and state his main results. We start with the definition of the inner core and the notion of competitive payoff vectors in the context of NTU market games. Afterwards, we state the main results of Qin (1993) and comment on the ideas he uses to prove them.

In order to define the inner core we first consider a game that is related to a compactly generated NTU game, called the $\lambda$-transfer game. Fix a transfer rate vector $\lambda \in \mathbb{R}_{+}^{n}$. 
Define $v_{\lambda}(S)=\max \{\lambda \cdot u \mid u \in V(S)\}$ as the maximal sum of weighted utilities that coalition $S$ can achieve given the transfer rate vector $\lambda$. The $\lambda$-transfer game, denoted as $\left(N, V_{\lambda}\right)$, of $(N, V)$ is defined by taking the same player set $N$ and the coalitional function $V_{\lambda}(S)=\left\{u \in \mathbb{R}^{S} \mid \lambda \cdot u \leq v_{\lambda}(S)\right\}$. Qin (1994, p.433) gives the following interpretation of the $\lambda$-transfer game: "The idea of the $\lambda$-transfer game may be captured by thinking of each player as representing a different country. The utilities are measured in different currencies, and the ratios $\lambda_{i} / \lambda_{j}$ are the exchange rates between the currencies of $i$ and $j$." As for the $\lambda$-transfer game only proportions matter we can assume without loss of generality that $\lambda$ is normalized, i.e. $\lambda \in \Delta=\left\{\lambda \in \mathbb{R}_{+}^{n} \mid \sum_{i=1}^{n} \lambda_{i}=1\right\}$. Define the positive unit simplex by $\Delta_{++}=\left\{\lambda \in \mathbb{R}_{++}^{n} \mid \sum_{i=1}^{n} \lambda_{i}=1\right\}$.

The inner core is a refinement of the core. The core $C(V)$ of an NTU game $(N, V)$ is defined as the set of utility allocations that are achievable by the grand coalition $N$ such that no coalition $S$ can improve upon this allocation. Thus,

$$
C(V)=\left\{u \in V(N) \mid \forall S \subseteq N \forall u^{\prime} \in V(S) \exists i \in S \text { such that } u_{i}^{\prime} \leq u_{i}\right\} \text {. }
$$

A utility allocation is in the inner core $I C(V)$ of a compactly generated game $(N, V)$ if it is achievable by the grand coalition $N$ and if additionally there exists a transfer rate vector $\lambda \in \Delta$ such that this utility allocation is in the core of the $\lambda$-transfer game. More precisely:

Definition (inner core). The inner core of a compactly generated NTU game $(N, V)$ is given by

$$
I C(V)=\left\{u \in V(N) \mid \exists \lambda \in \Delta \text { such that } u \in C\left(V_{\lambda}\right)\right\} .
$$

Qin (1993, Remark 1, p. 337) remarks that if the NTU game is compactly convexly generated the vectors of supporting weights for a utility vector in the inner core must all be strictly positive. This can be seen by the following argument: If for one player $i \in N \lambda_{i}$ is equal to 0 , then the core of the $\lambda$-transfer game is empty, because player $i$ can improve upon any $u \in V_{\lambda}(N)$ by forming the singleton coalition $\{i\}$.

Qin (1994) considers sufficient conditions for the inner core to be non-empty. In particular he shows that a compactly generated NTU game $(N, V)$, where $V(N)$ is convex, has a non-empty inner core if it is balanced with slack, meaning that for balancing weights $\left(\gamma_{S}\right)_{S \subseteq N}$ with $\gamma_{N}=0$ we have $\sum_{S \subset N} \gamma_{S} V(S) \subset \operatorname{int}_{\mathbb{R}^{n}} V(N)$ where $\operatorname{int}_{\mathbb{R}^{n}} V(N)$ is the interior of $V(N)$ relative to $\mathbb{R}^{n}$. Other contributions related to the non-emptiness of the inner core can be found for example in Iehlé (2004), Bonnisseau and Iehlé (2007) or Inoue (2010a).

We now define a competitive equilibrium for a market $\mathcal{E}$. 
Definition (competitive equilibrium). A competitive equilibrium for a market $\mathcal{E}$ is a tuple

$$
\left(\left(\hat{x}^{i}\right)_{i \in N},\left(\hat{y}^{i}\right)_{i \in N}, \hat{p}\right) \in \mathbb{R}_{+}^{\ell n} \times \mathbb{R}_{+}^{\ell n} \times \mathbb{R}_{+}^{\ell}
$$

such that

(i) $\sum_{i \in N} \hat{x}^{i}=\sum_{i \in N}\left(\hat{y}^{i}+\omega^{i}\right)$ (market clearing),

(ii) for all $i \in N, \hat{y}^{i}$ solves $\max _{y^{i} \in Y^{i}} \hat{p} \cdot y^{i}$ (profit maximization),

(iii) and for all $i \in N, \hat{x}^{i}$ is maximal with respect to the utility function $u^{i}$ in the budget set $\left\{x^{i} \in X^{i} \mid \hat{p} \cdot x^{i} \leq \hat{p} \cdot\left(\omega^{i}+\hat{y}^{i}\right)\right\}$ (utility maximization).

Given a competitive equilibrium its competitive payoff vector is defined as $\left(u^{i}\left(\hat{x}^{i}\right)\right)_{i \in N}$.

Qin (1993) investigates the relationship between the inner core of an NTU market game and the set of competitive payoff vectors of a market that represents this game. He establishes, following a conjecture of Shapley and Shubik (1975), the two theorems below analogously to the TU-case of Shapley and Shubik (1975).

Theorem (1, Qin (1993)). The inner core of an NTU market game coincides with the set of competitive payoff vectors of the induced market by that game.

Theorem (3, Qin (1993)). For every NTU market game and for any given point in its inner core, there is a market that represents the game and further has the given inner core point as its unique competitive payoff vector.

To show his first result Qin (1993) uses the notion of the induced market of a compactly convexly generated NTU game as it was already used by Billera and Bixby (1974). It turns out that the set of competitive equilibrium payoff vectors of the induced market coincides with the inner core. For his second result Qin (1993) fixes an inner core point, denoted by $u^{*} 1$, and chooses one transfer rate vector $\lambda_{u^{*}}^{*}$ from an associated $\lambda$-transfer game. He modifies the given NTU game by applying a suitable strictly monotonic transformation on the utility allocations a coalition can reach. In this modified game the given inner core point $u^{*}$ can be strictly separated from the set of utility allocations the grand coalition can reach (excluding $u^{*}$ ). Denote the modified game by $(N, \bar{V})$ and the convex compact sets generating this game by $\left(\bar{C}^{S}\right)_{S \in \mathcal{N}}$. A market to prove Theorem 3 of Qin (1993) can be defined as follows:

\footnotetext{
${ }^{1}$ Qin (1993) considers only NTU games where for all coalitions $S \in \mathcal{N}$ the generating sets satisfy $C^{S} \subseteq \mathbb{R}_{+}^{S}$ and $C^{S} \cap \mathbb{R}_{++}^{S} \neq \emptyset$ and hence has $u^{*} \gg 0$.
} 
Define for all coalitions $S \in \mathcal{N}$

$$
\begin{aligned}
& A_{S}^{1}=\left\{\left(u^{S},-e^{S},-e^{S},-e^{S}, 0\right) \mid u^{S} \in \bar{C}^{S}\right\} \subseteq \mathbb{R}^{5 n}, \\
& A_{S}^{2}=\left\{\left(u^{S}, 0,-e^{S}, 0,-e^{S}\right) \mid u^{S} \in \bar{C}^{S}\right\} \subseteq \mathbb{R}^{5 n} \\
& A_{S}^{3}=\left\{\left(u^{S}, 0,0,-e^{S},-e^{S}\right) \mid u^{S} \in \bar{C}^{S}\right\} \subseteq \mathbb{R}^{5 n} .
\end{aligned}
$$

Let $\mathcal{E}_{\bar{V}, u^{*}}=\left\{\left(X^{i}, Y^{i}, \omega^{i}, u^{i}\right)_{i \in N}\right\}$ be the market with for every individual $i \in N$

- the consumption set $X^{i}=X=\mathbb{R}_{+}^{n} \times\{(0,0,0)\} \times \mathbb{R}_{+}^{n} \subseteq \mathbb{R}_{+}^{5 n}$,

- the production set $Y^{i}=Y=$ convexcone $\left[\bigcup_{S \subseteq N}\left(A_{S}^{1} \cup A_{S}^{2} \cup A_{S}^{3}\right)\right] \subseteq \mathbb{R}^{5 n}$,

- the initial endowment vector $\omega^{i}=\left(0, e^{\{i\}}, e^{\{i\}}, e^{\{i\}}, e^{\{i\}}\right) \in \mathbb{R}_{+}^{5 n}$,

- the utility function $u^{i}\left(x^{i}\right)=\min \left\{x_{i}^{(1) i}, \frac{\left(\lambda_{u^{*}}^{*} \circ u^{*}\right) \cdot x^{(5) i}}{\lambda_{u^{*} i}^{*}}\right\}$ with $x^{i}=\left(x^{(1) i}, 0,0,0, x^{(5) i}\right) \in$ $X^{i}$ and $x_{k}^{(1) i}$ is the $k^{\text {th }}$ entry of $x^{(1) i}$.

Note that, similarly to the induced market, all individuals have the same consumption sets and the same production sets. The individuals differ in their initial endowment vectors and their utility functions. Qin (1993) introduces the sets $A_{S}^{1}, A_{S}^{2}, A_{S}^{3}$ in order to be able to show that the equilibrium price vector for the $5^{\text {th }}$ group of $n$ goods, $\hat{p}^{(5)}$, is strictly positive. The $i^{t h}$ consumer obtains utility from the $i^{\text {th }}$ component of the vector of the $1^{\text {st }}$ group of $n$ goods and from all the $5^{t h} n$ goods. The dependence of the utility function on all components of the $5^{\text {th }}$ group of $n$ goods is crucial to show the positiveness of $\hat{p}^{(5)}$. To prove his result Qin (1993) shows that the market $\mathcal{E}_{\bar{V}, u^{*}}$ represents the modified game and that the given inner core point is the unique competitive payoff vector of this economy. By applying the inverse strictly monotonic transformation to the utility functions he obtains his result.

In order to extend the results of Qin (1993) to a large class of closed subsets of the inner core we make use of the fact that for compactly convexly generated NTU games competitive payoff vectors need necessarily to be in the inner core. To see this we use a modified version of Proposition 1 from de Clippel and Minelli (2005).

Let $N=\{1, \ldots, n\}$ be the set of agents and $\{1, \ldots, \ell\}$ be the set of commodities. Let $X^{i} \subseteq \mathbb{R}_{+}^{\ell}$ be a convex set containing 0 , the consumption set of agent $i$. Each individual has a continuous, concave, (weakly) increasing and locally non-satiated utility function $u^{i}: \mathbb{R}_{+}^{\ell} \rightarrow \mathbb{R}$ and an initial endowment vector $\omega^{i} \in \mathbb{R}_{+}^{\ell} \backslash\{0\}$. Let $Y^{i} \subseteq \mathbb{R}^{\ell}$ be a non-empty and closed convex cone, the production set of agent $i$ 's firm. 
Lemma 1. Let $\left(\left(\hat{x}^{i}\right)_{i \in N},\left(\hat{y}^{i}\right)_{i \in N}, \hat{p}\right)$ be a competitive equilibrium such that $\hat{p} \cdot \omega^{i}>0$ for all individuals $i \in N$. Then $\left(u^{i}\left(\hat{x}^{i}\right)\right)_{i \in N}$ is in the inner core of the game induced by the economy.

The proof of Lemma 1 can be found in Appendix A.1.

\section{An extension of the Results of Qin (1993)}

In the above two theorems Qin (1993) considers on the one hand the whole inner core and on the other hand a single point in the inner core. In this section we extend the results of Qin (1993) by showing a similar result for closed subsets of the inner core. In the following we consider NTU market games and closed subsets of the inner core with certain properties. We want to ensure that for every point in a subset of the inner core, denoted by $A$, of a given NTU market game $(N, V)$ we can find a normal vector such that this point is strictly separated from the set $V(N)$ without the point by the hyperplane using this normal vector. If we assume that the individual rational part of $V(N)$ is strictly convex, then this property is satisfied. Moreover, we want to assume that this set of normal vectors, where each normal vector corresponds to one point of the set $A$, is bounded below by a strictly positive vector. This means that the exchange rates, represented by the normal vectors, within the set $A$ cannot be too extreme. We make the following definition:

Definition (strict positive separability). A pair $[(N, V), A]$ consisting of a compactly, convexly generated and totally balanced NTU game $(N, V)$ and a closed subset $A$ of its inner core satisfies strict positive separability [SPS] if the following condition holds:

There exists an $\varepsilon>0$ and a mapping $\lambda: A \rightarrow \Delta_{++}$, that associates to every point $x \in A$ a normal vector $\lambda(x)=\lambda^{x}$, such that

- every point $x \in A$ can be strictly separated from the set $V(N) \backslash\{x\}$ using this normal vector $\lambda^{x}$, i.e.

$$
\lambda^{x} \cdot x>\lambda^{x} \cdot y \quad \text { for all } y \in V(N) \backslash\{x\}
$$

- for all $x \in A$ every coordinate of the normal vector $\lambda^{x}$ is strictly greater than $\varepsilon$, i.e.

$$
\lambda_{i}^{x}>\varepsilon \quad \text { for all } i \in N
$$

For a pair $[(N, V), A]$ satisfying strict positive separability there might exist more than on mapping $\lambda$ and more than one $\varepsilon$. In the following we always consider one fixed 
mapping $\lambda$ together with one fixed $\varepsilon$ satisfying the conditions. Whenever $\lambda$ or $\varepsilon$ appear we mean the ones we fixed knowing that we might have chosen different ones.

The assumption of strict positive separability is not as restrictive as it might appear. It is satisfied for example if the individual rational part of $V(N)$ is strictly convex and $A$ is a closed subset of the interior of the inner core.

Note that from $\varepsilon<\lambda_{i}^{x}=\frac{\lambda_{i}^{x}}{1} \leq \frac{\lambda_{i}^{x}}{\lambda_{j}^{x}}$ it follows that

$$
\varepsilon<\min _{i, j \in N} \frac{\lambda_{i}^{x}}{\lambda_{j}^{x}} \quad \text { for all } \lambda^{x}, x \in A \text {. }
$$

Figure 1 illustrates the idea of strict positive separability with some examples. Assume that we have always two players and that the coalitional function is given by $V(\{1\})=$ $V(\{2\})=\{0\}-\mathbb{R}_{+}$and $V(\{1,2\})$ is given as indicated in Figure 1.
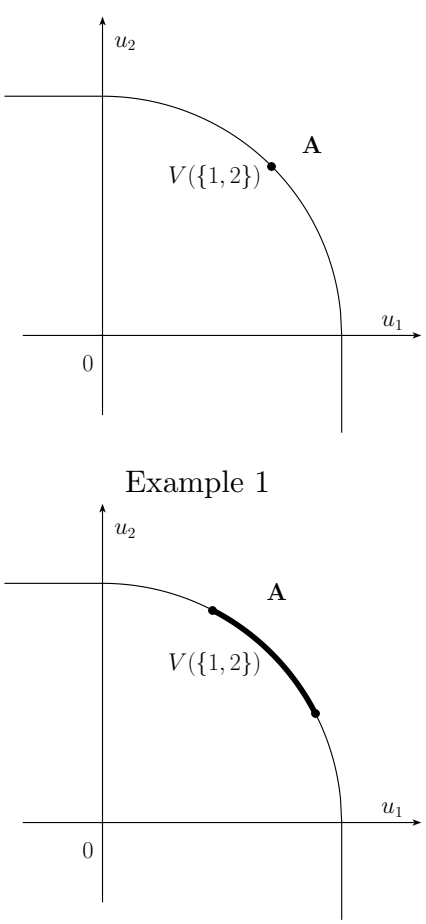

Example 4
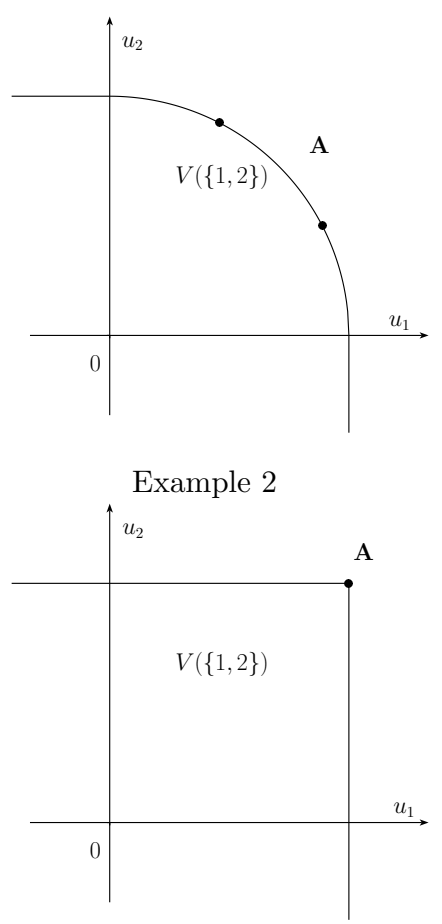

Example 5
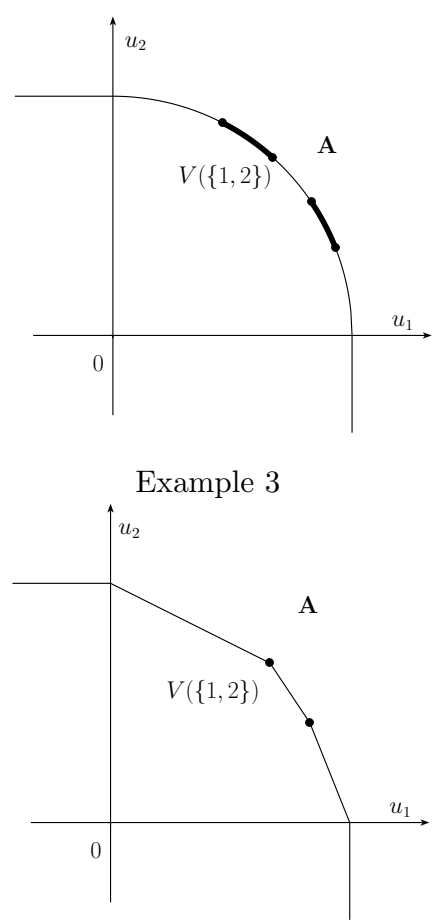

Example 6

Figure 1: Examples where SPS is satisfied. 
In Examples 1, 2, 3 and 4 the set $V(\{1,2\})$ is strictly convex. Here the inner core is given by all points on the efficient boundary without the two points on the axes. Thus, the NTU game together with every closed subset of its inner core satisfies SPS. This holds in particular for single points, finite sets, closed and connected sets or finite unions of closed sets.

Example 5 illustrates the case where the set $V(\{1,2\})$ is generated by a square and thus the inner core consists only of the corner point. In this case all the vectors in the strictly positive two-dimensional simplex support this inner core point. In order to establish SPS we just take one of these supporting vectors.

In Example 6 the set $V(\{1,2\})$ is generated by a polyhedron. The set $A$ is a finite set, consisting of some corner points of the polyhedron. For each of these corner points there exists a strictly positive normal vector that strictly separates it from $V(\{1,2\})$ without this corner point. The NTU game $(N, V)$ and this choice of the set $A$ satisfy SPS.

Figure 2 shows some examples that do not satisfy strict positive separability. As before assume that we have always two players and that the coalitional function is given by $V(\{1\})=V(\{2\})=\{0\}-\mathbb{R}_{+}$and $V(\{1,2\})$ is given as indicated in Figure 2.

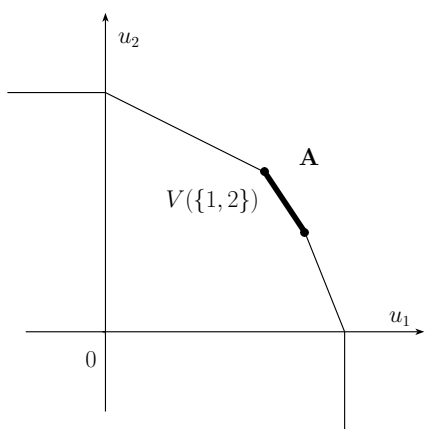

Example 7

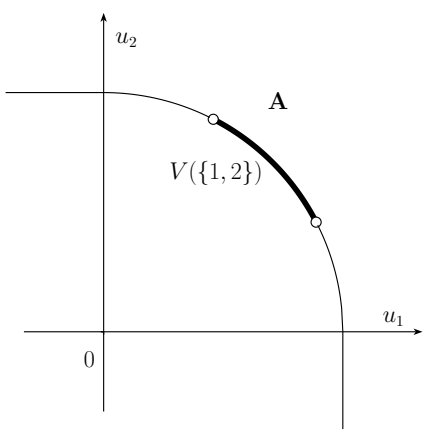

Example 8

Figure 2: Examples where SPS is not satisfied.

In contrast to Example 6, in Example 7 the set $A$ is chosen to be the line segment connecting two neighboring corner points of a polyhedron. Hence, all points in the set $A$ have a common normal vector. Thus, each of this points cannot be strictly separated from the polyhedron without this point. Therefore, SPS is not satisfied. In Example 8 each point in the set $A$ can be strictly separated from $V(\{1,2\})$ without the point. Nevertheless SPS is not satisfied, as the set $A$ is not closed. 
The properties, that we require at this point by considering only $[(N, V), A]$ satisfying SPS, are stronger than the properties, that we really need. For example it is sufficient if we can strictly separate each point in the boundary of $A$ from $A$ without it. Nevertheless, we choose to consider $[(N, V), A]$ which satisfy SPS, because they allow for an easy interpretation. After the presentation of the main results we discuss the question, how this can be weakened such that cases as in Example 6 are included in our results.

Now we prove the following result:

Theorem. Let $[(N, V), A]$ satisfy strict positive separability. Then there exists a market such that this market represents the game $(N, V)$ and such that the set of competitive payoff vectors of this market is the set $A$.

We show this result for NTU games where for every coalition $S \in \mathcal{N}$ we have $C^{S} \subseteq \mathbb{R}_{++}^{S}$. Due to the remark on page 6 this is not a restriction as we can shift an arbitrary given NTU game such that this condition is satisfied. After having applied our results we shift back the obtained economies such that they represent the original game. Hence, in the following if we consider an NTU game, we always assume for every coalition $S \in \mathcal{N}$ that we have $C^{S} \subseteq \mathbb{R}_{++}^{S}$.

Before beginning with the construction of a market satisfying the properties mentioned above, we introduce an auxiliary game and some notation.

Let $[(N, V), A]$ satisfy SPS. Let $(N, \tilde{V})$ be the NTU-game defined by

$$
\tilde{V}(S)=\left\{\begin{array}{cc}
V(S) & \text { if } S \subset N \\
\bigcap_{a \in A}\left\{z \in \mathbb{R}^{n} \mid \lambda^{a} \cdot z \leq \lambda^{a} \cdot a\right\} & \text { if } S=N
\end{array}\right.
$$

where $\lambda^{a}$ is as in the definition of SPS.

Note that to define the game $(N, \tilde{V})$ we use for every point of the set $a \in A$ just one normal vector that strictly separates this point from $V(N) \backslash\{a\}$. The games $(N, V)$ and $(N, \tilde{V})$ are equal except for the grand coalition $N$. For the coalition $N$ we extend the set $V(N)$ depending on the normal vectors of the set $A$. For illustration purposes figure 3 shows as an example for two players the sets $V(\{1,2\})$ and $\tilde{V}(\{1,2\})$.

To describe the relation between $(N, \tilde{V})$ and $(N, V)$ we introduce the following notation: Let $z \in \tilde{V}(N)$ and

$$
\bar{t}^{z}=\min \left\{t \in \mathbb{R}_{+} \mid z-t e^{N} \in V(N)\right\} .
$$

Define

$$
\tilde{C}^{N}=\left\{z \in \tilde{V}(N) \mid \exists t \in \mathbb{R}_{+} \text {such that } z-t e^{N} \in C^{N}\right\} .
$$




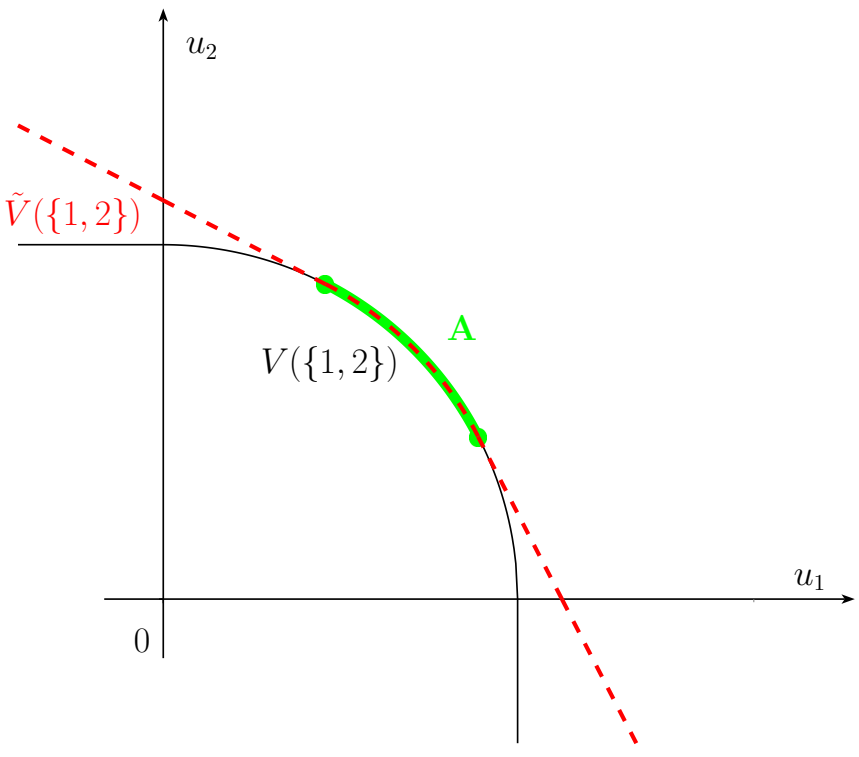

Figure 3: Example: The sets $V(\{1,2\})$ and $\tilde{V}(\{1,2\})$ for $N=\{1,2\}$.

Then we also have $\tilde{C}^{N}=\left\{z \in \tilde{V}(N) \mid z-\bar{t}^{z} e^{N} \in C^{N}\right\}$.

The following remark is easy to verify:

\section{Remark.}

1. The game $(N, V)$ is contained in the game $(N, \tilde{V})$. This means we have $V(S) \subseteq$ $\tilde{V}(S)$ for all $S \subseteq N$.

2. The set $\tilde{C}^{N}$ is convex and furthermore, $C^{N} \subseteq \tilde{C}^{N}$.

3. The game $(N, \tilde{V})$ is a convexly generated and totally balanced NTU-game, but it is not compactly generated. In particular we have $\tilde{V}(N) \neq \tilde{C}^{N}-\mathbb{R}_{+}^{n}$.

4. SPS ensures in particular: If we take $x$ in $V(N)$ outside from $A$, then $x$ is in the interior of $\tilde{V}(N)$,

$$
x \in V(N) \backslash A \Rightarrow x \in \operatorname{int}(\tilde{V}(N)) .
$$

The second point of the remark can be seen as follows: Take $z_{1}, z_{2} \in \tilde{C}^{N}$ and $\alpha \in[0,1]$. Then there exist $t^{z_{1}}$ and $t^{z_{2}}$ such that $z_{1}-t^{z_{1}} e^{N} \in C^{N}$ and $z_{2}-t^{z_{2}} e^{N} \in C^{N}$. As $C^{N}$ is per assumption convex $\alpha\left(z_{1}-t^{z_{1}} e^{N}\right)+(1-\alpha)\left(z_{2}-t^{z_{2}} e^{N}\right) \in C^{N}$. As well the set $\tilde{V}(N)$, as an intersection of halfspaces, is convex and hence $\alpha z_{1}+(1-\alpha) z_{2} \in \tilde{V}(N)$. Thus 
taking $t^{\alpha z_{1}+(1-\alpha) z_{2}}=\alpha t^{z_{1}}+(1-\alpha) t^{z_{2}}$ shows that $\left(\alpha z_{1}+(1-\alpha) z_{2}\right)-t^{\alpha z_{1}+(1-\alpha) z_{2}} e^{N}=$ $\alpha\left(z_{1}-t^{z_{1}} e^{N}\right)+(1-\alpha)\left(z_{2}-t^{z_{2}} e^{N}\right) \in C^{N}$. Therefore, we have $\alpha z_{1}+(1-\alpha) z_{2} \in \tilde{C}^{N}$. Hence, $\tilde{C}^{N}$ is convex.

Definition. Define the mapping $P_{A}: \tilde{V}(N) \longrightarrow V(N)$ via

$$
P_{A}(x)=x-\bar{t}^{x} e^{N} .
$$

The following figure illustrates the mapping $P_{A}$ for the example from figure 3 .

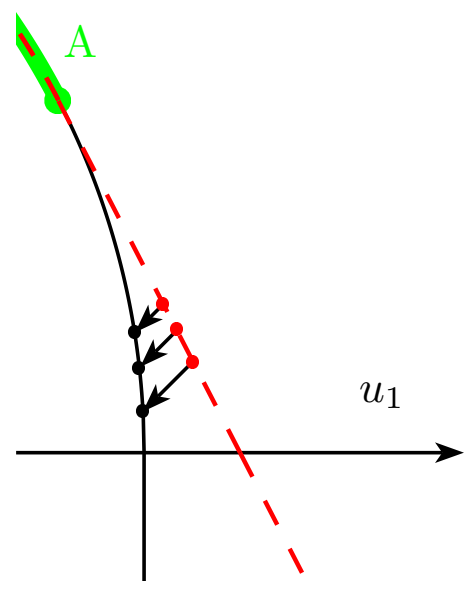

Figure 4: Illustration of the mapping $P_{A}$ for the example from figure 3 .

Note, that if $x \in V(N)$ then $\bar{t}^{x}=0$ and $P_{A}(x)=x$.

\section{Remark.}

1. The mapping $P_{A}$ is continuous and its image is $V(N)$.

2. The set $\tilde{C}^{N}$ can be written as

$$
\tilde{C}^{N}=\left\{z \in \tilde{V}(N) \mid P_{A}(z) \in C^{N}\right\}=P_{A}^{-1}\left(C^{N}\right),
$$

thus we have $P_{A}\left(\tilde{C}^{N}\right)=C^{N}$.

\subsection{The basic idea}

First, we present an intermediate result, which is interesting in itself. For $[(N, V), A]$ satisfying SPS we construct a market such that this market represents the given game and such that the set of payoff vectors of competitive equilibria with strictly positive 
price vectors coincides with the given set $A$. In the last chapter we show, how we deal with the case, when the equilibrium price vectors are not necessarily strictly positive, using a more complicated market with a similar structure.

Definition. Let $[(N, V), A]$ satisfy SPS. Then the market $\mathcal{E}_{V, A}^{0}$ is defined by

$$
\mathcal{E}_{V, A}^{0}=\left\{\left(X^{i}, Y^{i}, u^{i}, \omega^{i}\right)_{i \in N}\right\}
$$

with for every individual $i \in N$

- the consumption set $X^{i}=\mathbb{R}_{+}^{n} \times\{0\} \times \mathbb{R}_{+}^{n} \times\{0\} \subseteq \mathbb{R}^{4 n}$,

- the production set

$$
\begin{aligned}
Y^{i}=\text { convexcone } & {\left[\left(\bigcup_{S \in \mathcal{N} \backslash\{N\}, c^{S} \in C^{S}}\left(c^{S},-e^{S}, c^{S},-e^{S}\right)\right)\right.} \\
& \left.\cup\left(\bigcup_{\tilde{c}^{N} \in \tilde{C}^{N}}\left(P_{A}\left(\tilde{c}^{N}\right),-e^{N}, \tilde{c}^{N},-e^{N}\right)\right)\right] \subseteq \mathbb{R}^{4 n},
\end{aligned}
$$

- the initial endowment vector $\omega^{i}=\left(0, e^{\{i\}}, 0, e^{\{i\}}\right)$,

- and the utility function $u^{i}: X^{i} \rightarrow \mathbb{R}$ with $u^{i}\left(\left(x^{(1)}, 0, x^{(3)}, 0\right)\right)=\min \left(x_{i}^{(1)}, x_{i}^{(3)}\right)$.

Note that this market has the same consumption and production set for every individual $i \in N$. The individuals differ in their initial endowment vectors and their utility functions. There are input and output commodities. The $2^{\text {nd }}$ group and the $4^{\text {th }}$ group of $n$ commodities are the input commodities and every individual $i \in N$ owns one unit of his personal input commodity in the $i^{\text {th }}$ component of the $2^{\text {nd }}$ and the $4^{\text {th }}$ group of $n$ goods. The $1^{\text {st }}$ and the $3^{\text {rd }}$ group of $n$ goods are the output commodities, from whose $i^{t h}$ component player $i \in N$ obtains utility. The construction of this market is based on the idea of the induced market in Billera and Bixby (1974) or Qin (1993).

We now need to establish first that the market $\mathcal{E}_{V, A}^{0}$ is indeed a market for the NTU market game $(N, V)$.

Lemma 2. The market $\mathcal{E}_{V, A}^{0}$ represents the game $(N, V)$.

The proof of Lemma 2 is inspired by Billera (1974).

Proof.

- As $V(S)=C^{S}-\mathbb{R}_{+}^{S}$ it is enough to show, that for all $S \in \mathcal{N}$ the payoff vectors in the set $C^{S}$ can be achieved by coalition $S$ in the market $\mathcal{E}_{V, A}^{0}$. Let $z \in C^{S}$. 
We show, that there exists a feasible $S$-allocation $\left(x^{i}\right)_{i \in S}$ with $\left(y^{i}\right)_{i \in S}$ such that $u^{i}\left(x^{i}\right)=z_{i}$ for all $i \in S$.

Define for $i \in S$ the consumption plan

$$
x^{i}=\left(z^{\{i\}}, 0, z^{\{i\}}, 0\right)
$$

and let

$$
y^{i}=\frac{1}{|S|}\left(z,-e^{S}, z,-e^{S}\right)
$$

be the production plan for all $i \in S$. By the definition of the consumption sets we observe $x^{i} \in X^{i}$ for all $i \in S$. With regard to the production sets for $S \neq N$ we have immediately $y^{i} \in Y^{i}$ for all $i \in S$. For $S=N$ note that $z \in V(N) \subseteq \tilde{V}(N)$ and thus $P_{A}(z)=z$. Hence, we have $y^{i} \in Y^{i}$ for all $i \in N$. Observe that

$$
\sum_{i \in S}\left(x^{i}-\omega^{i}\right)=\sum_{i \in S} y^{i}
$$

Hence, $\left(x^{i}\right)_{i \in S}$ is a feasible $S$-allocation and

$$
u^{i}\left(x^{i}\right)=z_{i} \quad \text { for all } i \in S \text {. }
$$

- Let $\left(\bar{x}^{(1) i}, 0, \bar{x}^{(3) i}, 0\right)_{i \in S}$ be a feasible $S$-allocation with $\left(\bar{y}^{(1) i}, \bar{y}^{(2) i}, \bar{y}^{(3) i}, \bar{y}^{(4) i}\right)_{i \in S}$ in the market $\mathcal{E}_{V, A}^{0}$.

The feasibility implies

$$
\left(\sum_{i \in S} \bar{x}^{(1) i},-e^{S}, \sum_{i \in S} \bar{x}^{(3) i},-e^{S}\right)=\sum_{i \in S}\left(\bar{y}^{(1) i}, \bar{y}^{(2) i}, \bar{y}^{(3) i}, \bar{y}^{(4) i}\right) .
$$

Each production set is a convex cone of a union of convex sets. Hence, an arbitrary production plan can be written in the following way: Choose one suitable element from each of the convex sets and build a linear combination (with non-negative coefficients) of these elements. For the $1^{\text {st }}$ and the $2^{\text {nd }}$ group of $n$ commodities we obtain, that there exist $\alpha_{R}^{i} \in \mathbb{R}_{+}$for all $R \in \mathcal{N}, z_{R}^{i} \in C^{R}$ for all $R \in \mathcal{N} \backslash\{N\}$ and $\tilde{z}_{N}^{i} \in \tilde{C}^{N}$, such that

$$
\left(\bar{y}^{(1) i}, \bar{y}^{(2) i}\right)=\sum_{R \in \mathcal{N} \backslash\{N\}} \alpha_{R}^{i}\left(z_{R}^{i},-e^{R}\right)+\alpha_{N}^{i}\left(P_{A}\left(\tilde{z}_{N}^{i}\right),-e^{N}\right) .
$$

As $P_{A}\left(\tilde{C}^{N}\right)=C^{N}$ there exists $z_{N}^{i} \in C^{N}$ such that $P_{A}\left(\tilde{z}_{N}^{i}\right)=z_{N}^{i}$ and hence we 
have

$$
\left(\bar{y}^{(1) i}, \bar{y}^{(2) i}\right)=\sum_{R \in \mathcal{N}} \alpha_{R}^{i}\left(z_{R}^{i},-e^{R}\right) .
$$

As feasibility implies $\left(\sum_{i \in S} \bar{x}^{(1) i},-e^{S}\right)=\sum_{i \in S}\left(\bar{y}^{(1) i}, \bar{y}^{(2) i}\right)$, for the $2^{\text {nd }}$ group of $n$ coordinates we have that

$$
\begin{aligned}
e^{S} & =\sum_{i \in S} \sum_{R \in \mathcal{N}} \alpha_{R}^{i} e^{R} \\
& =\sum_{R \in \mathcal{N}}\left(\sum_{i \in S} \alpha_{R}^{i}\right) e^{R} .
\end{aligned}
$$

Thus $\alpha_{R}^{i}>0$ implies $R \subseteq S$ and if we define $\alpha(R)=\sum_{i \in S} \alpha_{R}^{i}$, then $(\alpha(R))_{R \subseteq S}$ is a balanced family for the coalition $S$. Looking at the $1^{\text {st }}$ group of $n$ coordinates we have

$$
\begin{aligned}
\sum_{i \in S} \bar{x}^{(1) i} & =\sum_{R \subseteq S} \sum_{i \in S} \alpha_{R}^{i} z_{R}^{i} \\
& =\sum_{\{R \subseteq S \mid \alpha(R)>0\}} \alpha(R)\left(\frac{1}{\alpha(R)} \sum_{i \in S} \alpha_{R}^{i} z_{R}^{i}\right) .
\end{aligned}
$$

Since $C^{R}$ is convex we have

$$
\frac{1}{\alpha(R)} \sum_{i \in S} \alpha_{R}^{i} z_{R} \in C^{R}
$$

and hence, using totally balancedness, $\sum_{i \in S} \bar{x}^{(1) i} \in V(S)$.

From the definition of the utility function we obtain $u^{i}\left(\bar{x}^{(1) i}, 0, \bar{x}^{(3) i}, 0\right) \leq \bar{x}_{i}^{(1) i}$. Since $\left(\bar{x}_{i}^{(1) i}\right)_{i \in S} \leq \sum_{i \in S} \bar{x}^{(1) i} \in V(S)$ we have by the $S$-comprehensiveness of $V(S)$ that $\left(u^{i}\left(\bar{x}^{(1) i}, 0, \bar{x}^{(3) i}, 0\right)\right)_{i \in S} \in V(S)$.

We verify that the payoff vectors in the set $A$ are indeed competitive payoff vectors of the market $\mathcal{E}_{V, A}^{0}$ :

Proposition 1. Every point in the set $A$ is equilibrium payoff vector of the market $\mathcal{E}_{V, A}^{0}$. 
Proof. Let $a \in A$ and $\lambda^{a} \in \Delta$ be a normal vector such that $a$ is in the core of the $\lambda^{a}$ transfer game. We know that $\lambda^{a}$ is strictly positive (compare the remark on page 8). By the assumption that $C^{N} \subseteq \mathbb{R}_{++}^{N}$ we know that $a$ is strictly positive. To prove the proposition, we show that the consumption and production plans

$$
\left(\hat{x}^{i}\right)_{i \in N}=\left(\left(a^{\{i\}}, 0, a^{\{i\}}, 0\right)\right)_{i \in N}
$$

and

$$
\left(\hat{y}^{i}\right)_{i \in N}=\left(\left(\frac{1}{n}\left(a,-e^{N}, a,-e^{N}\right)\right)\right)_{i \in N}
$$

together with the price system

$$
\hat{p}=\left(\lambda^{a}, \lambda^{a} \circ a, \lambda^{a}, \lambda^{a} \circ a\right)
$$

constitute a competitive equilibrium in the market $\mathcal{E}_{V, A}^{0}$.

First note that as $P_{A}(a)=a$ we have $\hat{y}^{i} \in Y^{i}$ for all $i \in N$. According to the remark above, the price system $\hat{p}$ is strictly positive. As we have a convex-cone-technology maximum profits are zero. We observe

$$
\hat{p} \cdot \hat{y}^{i}=\frac{1}{n}\left(\lambda^{a} \cdot a-\left(\lambda^{a} \circ a\right) \cdot e^{N}+\lambda^{a} \cdot a-\left(\lambda^{a} \circ a\right) \cdot e^{N}\right)=0 .
$$

Hence, the production plan $\hat{y}^{i}$ is profit maximizing.

As we have a min-type or Leontief utility function, it is optimal for each agent $i$ to spend his budget in a way such that $\hat{x}_{i}^{(1) i}=\hat{x}_{i}^{(3) i}$ and that he does not consume anything of the other commodities. Furthermore, he has to spend all his budget, because the preferences are locally non-satiated and continuous. The budget constraint is satisfied with equality,

$$
\hat{p} \cdot \hat{x}^{i}=\lambda^{a} \cdot\left(a^{\{i\}}+a^{\{i\}}\right)=\left(\lambda^{a} \circ a\right) \cdot\left(e^{\{i\}}+e^{\{i\}}\right)=\hat{p} \cdot \omega^{i}
$$

and

$$
\hat{x}^{(1) i}=a^{\{i\}}=\hat{x}^{(3) i} .
$$

Hence, the consumption vector $\hat{x}^{i}$ is utility maximizing on the budget set of agent $i$.

Furthermore, the market clearing condition

$$
\sum_{i \in N} \hat{x}^{i}=\sum_{i \in N} \omega^{i}+\sum_{i \in N} \hat{y}^{i}
$$

is satisfied. 
Thus, we have found a competitive equilibrium with equilibrium payoff vector

$$
\left(u^{i}\left(\hat{x}^{i}\right)\right)_{i \in N}=a .
$$

Looking again at the competitive equilibrium price vectors in the proof of Proposition 1 note: For a competitive equilibrium with payoff vector $a \in A$ the equilibrium price vector for the $1^{\text {st }}$ (respectively $3^{\text {rd }}$ ) group of $n$ goods, the output goods, is the normal vector $\lambda^{a}$ separating the point $a$ from $V(N)$. The transfer rate vectors coincide with the equilibrium prices for the output goods of the market. The input goods are priced by $\lambda^{a} \circ a$. This is the transfer rate vector weighted by the according point of the set $A$. Interpreted differently: The input goods are first weighted by the point $a$ of the set $A$ and afterwards they are priced by the transfer rate vector $\lambda^{a}$. The relationship of the transfer rate vectors and the prices of competitive equilibria was observed in several publications discussing the relation between NTU games and economies. Examples are Shubik (1985), Shapley (1987), Trockel (1996) and Qin (1993). Shapley (1987, p. 192) states: "There is a strong analogy though no formal equivalence that we know of between the comparison weights that we must introduce in order to obtain a feasible transfer value and the prices in a competitive market." Here we obtain a formal equivalence for the prices of the output goods and an indirect link for the prices of the input goods. Trockel (1996) investigated this equivalence for NTU bargaining games and Qin (1993) obtained very similar equilibrium prices as we have here.

Next, we consider the utility allocations outside the set $A$. Using Lemma 1 it is sufficient to consider those vectors in the inner core.

Proposition 2. Any payoff vector of a competitive equilibrium of the market $\mathcal{E}_{V, A}^{0}$ with a strictly positive equilibrium price vector is an element of the set $A$.

Proof. Lemma 1 ensures that every competitive equilibrium payoff vector is in the inner core. Assume that there exists a competitive equilibrium $\left(\left(x^{i}\right)_{i \in N},\left(y^{i}\right)_{i \in N}, p\right)$ such that its payoff vector $\left(u^{i}\left(x^{i}\right)\right)_{i \in N}$ is in the inner core but not in the set $A$ and such that the equilibrium price vector is strictly positive, $p \gg 0$.

Then, there exists an element $c^{N}$ in the inner core outside $A$ such that $u^{i}\left(x^{i}\right)=c_{i}^{N}$ for all player $i=1, \ldots, n$. Let $x^{i}=\left(x^{(1) i}, x^{(2) i}, x^{(3) i}, x^{(4) i}\right)$. By the definition of the consumption set we know $x^{(2) i}=x^{(4) i}=0$ and by the definition of the utility function we obtain $x_{i}^{(1) i} \geq c_{i}^{N}$ and $x_{i}^{(3) i} \geq c_{i}^{N}$ for all $i=1, \ldots, n$. 
Claim 1: From the utility maximization and the strict positivity of the price vector it follows that we need to have

$$
x_{i}^{(1) i}=c_{i}^{N}=x_{i}^{(3) i} .
$$

The proof of Claim 1 can be found in Appendix A.2.

We get by the market clearing condition: $y=\sum_{i \in N}\left(x^{i}-\omega^{i}\right)=\left(c^{N},-e^{N}, c^{N},-e^{N}\right)$. But the production plan $y=\left(c^{N},-e^{N}, c^{N},-e^{N}\right)$ is not profit maximizing. ${ }^{2}$

To see this notice the following: As $c^{N}$ is in the inner core but outside the set $A$ there exists a $\tilde{c}^{N}$ with $P_{A}\left(\tilde{c}^{N}\right)=c^{N}$ and $\tilde{c}^{N} \gg c^{N}$. Consider the production plan $\left(P_{A}\left(\tilde{c}^{N}\right),-e^{N}, \tilde{c}^{N},-e^{N}\right)$. Looking at the profits and using the strict positivity of the price vector we observe

$$
\begin{aligned}
p \cdot y & =p^{(1)} \cdot c^{N}-p^{(2)} \cdot e^{N}+p^{(3)} \cdot c^{N}-p^{(4)} \cdot e^{N} \\
& <p^{(1)} \cdot c^{N}-p^{(2)} \cdot e^{N}+p^{(3)} \cdot \tilde{c}^{N}-p^{(4)} \cdot e^{N} \\
& =p^{(1)} \cdot P_{A}\left(\tilde{c}^{N}\right)-p^{(2)} \cdot e^{N}+p^{(3)} \cdot \tilde{c}^{N}-p^{(4)} \cdot e^{N} \\
& \leq 0 .
\end{aligned}
$$

Thus, we have found a production plan that has strictly higher profits than $y$. This is a contradiction, since $y$ needs to be profit maximizing.

It follows that with strictly positive price vectors the allocations outside the set $A$ but in the inner core cannot be competitive equilibrium payoff vectors.

Combining the two propositions above we obtain the following theorem:

Theorem. Let $[(N, V), A]$ satisfy strict positive separability. The set of payoff vectors of competitive equilibria with a strictly positive equilibrium price vector of the market $\mathcal{E}_{V, A}^{0}$ coincides with the set $A$.

\section{Positive equilibrium price vectors are required to obtain the above results}

Up to now we always considered competitive equilibria with only strictly positive equilibrium price vectors. This was indeed necessary. If we also allow for price vectors that are not strictly positive, then we can construct a competitive equilibrium with competitive payoff vectors outside the given set $A$. To see this fix $a \notin A$ but in the inner core. Then there exists $\tilde{a} \in \tilde{C}^{N}$ such that $P_{A}(\tilde{a})=a$ and $\tilde{a} \gg a$. Consider

\footnotetext{
${ }^{2}$ Since the individual production sets are convex cones, to check profit maximization it is sufficient to consider the joint production plans. We have $\sum_{i=1}^{n} Y^{i}=Y^{j}$ for any $j \in N$.
} 


$$
\begin{aligned}
\hat{x}^{i} & =\left(\left(P_{A}(\tilde{a})\right)^{\{i\}}, 0, \tilde{a}^{\{i\}}, 0\right)=\left(a^{\{i\}}, 0, \tilde{a}^{\{i\}}, 0\right) \text { for all } i \in N, \\
\hat{y}^{i} & =\left(\frac{1}{n}\left(P_{A}(\tilde{a}),-e^{N}, \tilde{a},-e^{N}\right)\right)=\left(\frac{1}{n}\left(a,-e^{N}, \tilde{a},-e^{N}\right)\right) \text { for all } i \in N, \\
\hat{p} & =\left(\lambda^{a}, \lambda^{a} \circ a, 0,0\right)
\end{aligned}
$$

where $\lambda^{a}$ is one normal vector from a $\lambda^{a}$-transfer game and $\left(P_{A}(\tilde{a})\right)^{\{i\}}$ is the vector that has as its $i^{t h}$ coordinate the $i^{t h}$ coordinate of $P_{A}(\tilde{a})$ and zero coordinates otherwise. Analogously define $\tilde{a}^{\{i\}}$.

We show that $\left(\left(\hat{x}^{i}\right)_{i \in N},\left(\hat{y}^{i}\right)_{i \in N}, \hat{p}\right)$ constitutes a competitive equilibrium with the payoff vector $a \notin A$.

- First note that $u^{i}\left(\hat{x}^{i}\right)=\min \left\{a_{i}, \tilde{a}_{i}\right\}=a_{i}$, since we have $\tilde{a} \gg a$.

- For the profit maximization we obtain

$$
\hat{p} \cdot \hat{y}^{i}=\frac{1}{n}\left(\lambda^{a} \cdot a-\left(\lambda^{a} \circ a\right) \cdot e^{N}\right)=0 .
$$

Since the maximum profits are zero, $\hat{y}^{i}$ is profit maximizing.

- For the utility maximization we obtain that the budget constraint is satisfied with equality,

$$
\hat{p} \cdot \hat{x}^{i}=\lambda^{a} \cdot a^{\{i\}}=\left(\lambda^{a} \circ a\right) \cdot e^{\{i\}}=\hat{p} \cdot \omega^{i},
$$

and furthermore individual $i$ spends all his budget for the $i^{\text {th }}$ commodity in the $1^{\text {st }}$ group of $n$ goods. Since the prices are equal to zero for the $3^{r d}$ and $4^{\text {th }}$ group of $n$ goods he can consume $\hat{x}_{i}^{(3) i}=\tilde{a}_{i}$ without using any of his budget. Thus, $\hat{x}^{i}$ is utility maximizing.

- Moreover, the market clearing condition is satisfied

$$
\sum_{i \in N} \hat{x}^{i}=\sum_{i \in N} \omega^{i}+\sum_{i \in N} \hat{y}^{i} .
$$

Thus, we have found a competitive equilibrium with equilibrium payoff vector

$$
\left(u^{i}\left(\hat{x}^{i}\right)\right)_{i \in N}=a \notin A .
$$




\subsection{The main results}

In order to deal with the general case without assuming the strict positivity of price vectors, we modify the market from the previous section in an appropriate way. This modification allows us to show, that the prices of the $3^{r d}$ group of $n$ commodities are strictly positive, $p^{(3)} \gg 0$. For the rest of this section let $[(N, V), A]$ satisfy SPS. To simplify the notation of the market, we introduce some sets before:

For the definition of the production sets define for all coalitions $S \in \mathcal{N} \backslash\{N\}$

$$
\begin{aligned}
& A_{S}^{1}=\left\{\left(c^{S},-e^{S}, c^{S},-e^{S},-e^{S}\right) \mid c^{S} \in C^{S}\right\}, \\
& A_{S}^{2}=\left\{\left(c^{S}, 0, c^{S},-e^{S}, 0\right) \mid c^{S} \in C^{S}\right\}, \\
& A_{S}^{3}=\left\{\left(c^{S}, 0, c^{S}, 0,-e^{S}\right) \mid c^{S} \in C^{S}\right\}
\end{aligned}
$$

and for the grand coalition $N$ define

$$
\begin{aligned}
& A_{N}^{1}=\left\{\left(P_{A}\left(\tilde{c}^{N}\right),-e^{N}, \tilde{c}^{N},-e^{N},-e^{N}\right) \mid \tilde{c}^{N} \in \tilde{C}^{N}\right\}, \\
& A_{N}^{2}=\left\{\left(P_{A}\left(\tilde{c}^{N}\right), 0, \tilde{c}^{N},-e^{N}, 0\right) \mid \tilde{c}^{N} \in \tilde{C}^{N}\right\}, \\
& A_{N}^{3}=\left\{\left(P_{A}\left(\tilde{c}^{N}\right), 0, \tilde{c}^{N}, 0,-e^{N}\right) \mid \tilde{c}^{N} \in \tilde{C}^{N}\right\} .
\end{aligned}
$$

In order to obtain the result without the assumption of strictly positive price vectors, we modify the utility functions, the production and consumption sets. The utility functions do not depend anymore only on the two personal output commodities but also on the whole second group of output commodities. For that we add 'a little bit' of utility from the other players output goods. This 'little bit' is described by using the $\varepsilon>0$ from the definition of SPS.

Definition (induced $A$-market). Let $[(N, V), A]$ satisfy strict positive separability. Let $\varepsilon>0$ such that $\varepsilon<\min _{i, j \in N} \frac{\lambda_{i}^{a}}{\lambda_{j}^{a}}$ for all $a \in A$. The induced $A$-market of the game $(N, V)$ and the set $A$ is defined by

$$
\mathcal{E}_{V, A, \varepsilon}=\left\{\left(X^{i}, Y^{i}, u^{i}, \omega^{i}\right)_{i \in N}\right\}
$$

with for every individual $i \in N$

- the consumption set $X^{i}=\mathbb{R}_{+}^{n} \times\{0\} \times \mathbb{R}_{+}^{n} \times\{0\} \times\{0\} \subseteq \mathbb{R}^{5 n}$,

- the production set $Y^{i}=$ convexcone $\left[\bigcup_{S \in \mathcal{N}}\left(A_{S}^{1} \cup A_{S}^{2} \cup A_{S}^{3}\right)\right] \subseteq \mathbb{R}^{5 n}$

- the initial endowment vector $\omega^{i}=\left(0, e^{\{i\}}, 0, e^{\{i\}}, e^{\{i\}}\right)$, 
- and the utility function $u^{i}: X^{i} \rightarrow \mathbb{R}$ with

$$
u^{i}\left(x^{(1)}, 0, x^{(3)}, 0,0\right)=\min \left(x_{i}^{(1)}, x_{i}^{(3)}+\varepsilon \sum_{j \neq i} x_{j}^{(3)}\right) .
$$

Note that this market is very similar to the market we defined in the previous section. We change the definition of the production and consumption sets slightly by introducing a further input commodity. Moreover, the utility functions here depend on all coordinates of the $3^{\text {rd }}$ group of $n$ goods.

Having defined the induced $A$-market we prove the following theorem, which is the main result of this paper:

Theorem. Let $[(N, V), A]$ satisfy strict positive separability. Then there exists a market such that this market represents the game $(N, V)$ and such that the set of competitive payoff vectors of this market is the set $A$.

To prove the above theorem we use the induced $A$-market $\mathcal{E}_{V, A, \varepsilon}$ as defined before. We divide the proof of this Theorem into 3 parts: First we show, that $\mathcal{E}_{V, A, \varepsilon}$ represents the game $(N, V)$, in the second part we prove, that every vector in the set $A$ is a competitive payoff vector, and in the third part we show that competitive payoff vectors always belong to the set $A$.

Lemma 3. The induced $A$-market $\mathcal{E}_{V, A, \varepsilon}$ represents the game $(N, V)$.

The proof of Lemma 3 is inspired by Billera (1974).

Proof.

- As $V(S)=C^{S}-\mathbb{R}_{+}^{S}$ it is enough to show, that the payoffs in the set $C^{S}$ can be achieved by coalition $S$ in the market $\mathcal{E}_{V, A, \varepsilon}$. Let $z \in C^{S}$. We show, that there exists a feasible $S$-allocation $\left(x^{i}\right)_{i \in S}$ with $\left(y^{i}\right)_{i \in S}$ such that $u^{i}\left(x^{i}\right)=z_{i}$ for all $i \in S$.

Define for $i \in S$ the consumption plan

$$
x^{i}=\left(z^{\{i\}}, 0, z^{\{i\}}, 0,0\right)
$$

and let

$$
y^{i}=\frac{1}{|S|}\left(z,-e^{S}, z,-e^{S},-e^{S}\right)
$$


be the production plan for all $i \in S$. By the definition of the consumption sets we observe $x^{i} \in X^{i}$ for all $i \in S$. With regard to the production sets for $S \neq N$ we have immediately $y^{i} \in Y^{i}$ for all $i \in S$. For $S=N$ note that $z \in V(N) \subseteq \tilde{V}(N)$ and thus $P_{A}(z)=z$. Hence, we have $y^{i} \in Y^{i}$ for all $i \in N$. Observe that

$$
\sum_{i \in S}\left(x^{i}-\omega^{i}\right)=\sum_{i \in S} y^{i}
$$

Hence, $\left(x^{i}\right)_{i \in S}$ is a feasible $S$-allocation and

$$
u^{i}\left(x^{i}\right)=z_{i} \quad \text { for all } i \in S \text {. }
$$

- Let $\left(\bar{x}^{(1) i}, 0, \bar{x}^{(3) i}, 0,0\right)_{i \in S}$ be a feasible $S$-allocation with $\left(\bar{y}^{(1) i}, \bar{y}^{(2) i}, \bar{y}^{(3) i}, \bar{y}^{(4) i}, \bar{y}^{(5) i}\right)_{i \in S}$ in the market $\mathcal{E}_{V, A, \varepsilon}$.

The feasibility implies

$$
\left(\sum_{i \in S} \bar{x}^{(1) i},-e^{S}, \sum_{i \in S} \bar{x}^{(3) i},-e^{S},-e^{S}\right)=\sum_{i \in S}\left(\bar{y}^{(1) i}, \bar{y}^{(2) i}, \bar{y}^{(3) i}, \bar{y}^{(4) i}, \bar{y}^{(5) i}\right) .
$$

Each production set is a convex cone of a union of convex sets. Hence, an arbitrary production plan can be written in the following way: Choose one suitable element from each of the convex sets and build a linear combination (with non-negative coefficients) of these elements. For the $1^{\text {st }}$ and the $2^{\text {nd }}$ group of $n$ commodities we obtain, that there exist $\alpha_{R}^{i} \in \mathbb{R}_{+}$for all $R \in \mathcal{N}, z_{R}^{i} \in C^{R}$ for all $R \in \mathcal{N} \backslash\{N\}$ and $\tilde{z}_{N}^{i} \in \tilde{C}^{N}$, such that

$$
\left(\bar{y}^{(1) i}, \bar{y}^{(2) i}\right)=\sum_{R \in \mathcal{N} \backslash\{N\}} \alpha_{R}^{i}\left(z_{R}^{i},-e^{R}\right)+\alpha_{N}^{i}\left(P_{A}\left(\tilde{z}_{N}^{i}\right),-e^{N}\right) .
$$

As $P_{A}\left(\tilde{C}^{N}\right)=C^{N}$ there exists $z_{N}^{i} \in C^{N}$ such that $P_{A}\left(\tilde{z}_{N}^{i}\right)=z_{N}^{i}$ and hence we have

$$
\left(\bar{y}^{(1) i}, \bar{y}^{(2) i}\right)=\sum_{R \in \mathcal{N}} \alpha_{R}^{i}\left(z_{R}^{i},-e^{R}\right) .
$$

As feasibility implies $\left(\sum_{i \in S} \bar{x}^{(1) i},-e^{S}\right)=\sum_{i \in S}\left(\bar{y}^{(1) i}, \bar{y}^{(2) i}\right)$, for the $2^{\text {nd }}$ group of $n$ coordinates we have that

$$
e^{S}=\sum_{i \in S} \sum_{R \in \mathcal{N}} \alpha_{R}^{i} e^{R}
$$




$$
=\sum_{R \in \mathcal{N}}\left(\sum_{i \in S} \alpha_{R}^{i}\right) e^{R} .
$$

Thus $\alpha_{R}^{i}>0$ implies $R \subseteq S$ and if we define $\alpha(R)=\sum_{i \in S} \alpha_{R}^{i}$, then $(\alpha(R))_{R \subseteq S}$ is a balanced family for the coalition $S$. Looking at the $1^{\text {st }}$ group of $n$ coordinates we have

$$
\begin{aligned}
\sum_{i \in S} \bar{x}^{(1) i} & =\sum_{R \subseteq S} \sum_{i \in S} \alpha_{R}^{i} z_{R}^{i} \\
& =\sum_{\{R \subseteq S \mid \alpha(R)>0\}} \alpha(R)\left(\frac{1}{\alpha(R)} \sum_{i \in S} \alpha_{R}^{i} z_{R}^{i}\right) .
\end{aligned}
$$

Since $C^{R}$ is convex we have

$$
\frac{1}{\alpha(R)} \sum_{i \in S} \alpha_{R}^{i} z_{R} \in C^{R}
$$

and hence, using totally balancedness, $\sum_{i \in S} \bar{x}^{(1) i} \in V(S)$.

From the definition of the utility function we obtain $u^{i}\left(\bar{x}^{(1) i}, 0, \bar{x}^{(3) i}, 0,0\right) \leq \bar{x}_{i}^{(1) i}$. Since $\left(\bar{x}_{i}^{(1) i}\right)_{i \in S} \leq \sum_{i \in S} \bar{x}^{(1) i} \in V(S)$ we have by the $S$-comprehensiveness of $V(S)$ that $\left(u^{i}\left(\bar{x}^{(1) i}, 0, \bar{x}^{(3) i}, 0,0\right)\right)_{i \in S} \in V(S)$.

Proposition 3. Every point in $A$ is an equilibrium payoff vector of the market $\mathcal{E}_{V, A, \varepsilon}$.

Proof. The above proposition holds by an argument similar to the one used in the proof of Proposition 1. Let $a \in A$ and $\lambda^{a} \in \Delta$ an associated normal vector. We know that $\lambda^{a}$ is strictly positive (compare the remark on page 8). Note that the consumption and production plans

$$
\left(\hat{x}^{i}\right)_{i \in N}=\left(\left(a^{\{i\}}, 0, a^{\{i\}}, 0,0\right)\right)_{i \in N}
$$

and

$$
\left(\hat{y}^{i}\right)_{i \in N}=\left(\left(\frac{1}{n}\left(a,-e^{N}, a,-e^{N},-e^{N}\right)\right)\right)_{i \in N}
$$

together with the price system

$$
\hat{p}=\left(\lambda^{a}, \frac{2}{3}\left(\lambda^{a} \circ a\right), \lambda^{a}, \frac{2}{3}\left(\lambda^{a} \circ a\right), \frac{2}{3}\left(\lambda^{a} \circ a\right)\right)
$$


constitute a competitive equilibrium in the market $\mathcal{E}_{V, A, \varepsilon}$. The equilibrium price vector is strictly positive since $a$ and $\lambda^{a}$ are strictly positive.

As we have a convex-cone-technology maximum profits are zero. We observe

$$
\hat{p} \cdot \hat{y}^{i}=\frac{1}{n}\left(\lambda^{a} \cdot a-\frac{2}{3}\left(\lambda^{a} \circ a\right) \cdot e^{N}+\lambda^{a} \cdot a-\frac{2}{3}\left(\lambda^{a} \circ a\right) \cdot e^{N}-\frac{2}{3}\left(\lambda^{a} \circ a\right) \cdot e^{N}\right)=0 .
$$

Hence, the production plan $\hat{y}^{i}$ is profit maximizing.

Next we show that the consumption vector $x^{i}$ is utility maximizing on the budget set of agent $i$.

- First notice that the budget constraint is satisfied with equality,

$$
\hat{p} \cdot \hat{x}^{i}=\lambda^{a} \cdot\left(a^{\{i\}}+a^{\{i\}}\right)=\frac{2}{3}\left(\lambda^{a} \circ a\right) \cdot\left(e^{\{i\}}+e^{\{i\}}+e^{\{i\}}\right)=\hat{p} \cdot \omega^{i} .
$$

- Second the consumption vector of agent $i$ satisfies

$$
\hat{x}_{i}^{(1) i}=\hat{x}_{i}^{(3) i}+\varepsilon \sum_{j \neq i} \hat{x}_{j}^{(3) i}
$$

This means agent $i$ consumes in a way such that he receives the "same amount of utility" from the $1^{\text {st }}$ group of $n$ goods and the $3^{r d}$ group of $n$ goods. For an agent with a min-type or Leontief utility function it is a necessary condition for utility maximization to consume in such a way (as long as we have strictly positive prices). This can be seen by similar arguments like in the proof of Claim 1.

- Third, it remains to check that $\hat{x}^{i}$ is indeed utility maximizing for agent $i$ on his budget set. Hereby, the crucial point to see is, that agent $i$ only consumes his personal output goods, and not the output goods of the other agents. In particular, this means for the $3^{\text {rd }}$ group of $n$ commodities $\hat{x}_{j}^{(3) i}=0$ for $j \neq i$.

First look at the consumption of the $3^{\text {rd }}$ group of $n$ goods when half of the wealth, $\lambda^{a} \cdot a^{\{i\}}$, is used for these goods.

If agent $i$ spends the wealth only for his personal output commodity, he consumes $\hat{x}^{(3) i}=a^{\{i\}}$. Then we have $\hat{p}^{(3)} \cdot \hat{x}^{(3) i}=\lambda^{a} \cdot a^{\{i\}}$. Suppose now agent $i$ changes his consumption plan for the $3^{\text {rd }}$ group of $n$ commodities to a plan $\tilde{x}^{(3) i}$, where he consumes as well one of the other agents output goods, meaning $\tilde{x}_{j}^{(3) i}>0$ for one $j \neq i$. To do this agent $i$ needs to decrease the consumption in his personal output good and hence $\hat{x}_{i}^{(3) i}>\tilde{x}_{i}^{(3) i}$. Set $\delta=\hat{x}_{i}^{(3) i}-\tilde{x}_{i}^{(3) i}$. Then this $\delta$ he consumes less gives him an available budget of $\lambda_{i}^{a} \delta$, that he can now use to spend for the other agents commodity $j$. If agent $i$ now spends $\lambda_{i}^{a} \delta$ for good $j$, he can purchase $\frac{\lambda_{i}^{a}}{\lambda_{j}^{a}} \delta$ 
units of good $j$ which gives him an additional level of "utility" in good $j$ of the $3^{\text {rd }}$ group of $n$ goods.

Look at

$$
\begin{aligned}
\hat{x}_{i}^{(3) i} & +\varepsilon \sum_{j \neq i} \hat{x}_{j}^{(3) i}-\left(\tilde{x}_{i}^{(3) i}+\varepsilon \sum_{j \neq i} \tilde{x}_{j}^{(3) i}\right) \\
& =\hat{x}_{i}^{(3) i}-\left(\hat{x}_{i}^{(3) i}-\delta+\varepsilon \frac{\lambda_{i}^{a}}{\lambda_{j}^{a}} \cdot \delta\right) \\
& =\delta-\varepsilon \frac{\lambda_{i}^{a}}{\lambda_{j}^{a}} \cdot \delta \\
& =\delta\left(1-\varepsilon \frac{\lambda_{i}^{a}}{\lambda_{j}^{a}}\right) .
\end{aligned}
$$

The above expression is positive since $\varepsilon<\frac{\lambda_{j}^{a}}{\lambda_{i}^{a}}$ for all $i, j \in N$ and hence $\varepsilon \frac{\lambda_{i}^{a}}{\lambda_{j}^{a}}<$ $\frac{\lambda_{j}^{a}}{\lambda_{i}^{a}} \frac{\lambda_{i}^{a}}{\lambda_{j}^{a}}=1$. Thus we have

$$
\hat{x}_{i}^{(3) i}+\varepsilon \sum_{j \neq i} \hat{x}_{j}^{(3) i}>\tilde{x}_{i}^{(3) i}+\varepsilon \sum_{j \neq i} \tilde{x}_{j}^{(3) i} .
$$

The potential loss of utility from consuming less of his personal output commodity is higher than the potential gain from consuming agent $j$ 's output commodity given a fixed wealth.

A similar argument also holds true, when agent $i$ changes the consumption in a way such that he consumes output goods of several other agents.

Thus agent $i$ cannot increase his utility by changing his consumption plan for the $3^{\text {rd }}$ group of $n$ commodities from $\hat{x}^{(3) i}$ to $\tilde{x}^{(3) i}$ and consuming output commodities of the other agents $j \neq i$ instead of his own output commodities.

Now it is easy to see, that spending half of the total wealth for each of the two groups of output commodities leads to the same amount of utility in both arguments of the min-type utility function and is hence utility maximizing.

Furthermore, the market clearing condition

$$
\sum_{i \in N} \hat{x}^{i}=\sum_{i \in N} \omega^{i}+\sum_{i \in N} \hat{y}^{i}
$$

is satisfied. 
Thus, we have found a competitive equilibrium with equilibrium payoff vector

$$
\left(u^{i}\left(\hat{x}^{i}\right)\right)_{i \in N}=a .
$$

In the above proof the competitive equilibrium price vectors are linked to the transfer rate vectors of points in the set $A$ similarly as in the proof of Proposition 1 . The output goods are directly priced by the transfer rate vectors and the input goods are priced by the transfer rate vectors weighted by the according point of the set $A$ (multiplied by $\frac{2}{3}$ ).

It remains to show, that vectors not belonging to the set $A$ cannot be competitive payoff vectors. The crucial point is to show, that $p^{(3)}$ is strictly positive.

Lemma 4. Let $\left(\left(x^{i}\right)_{i \in N},\left(y^{i}\right)_{i \in N}, p\right)$ be any competitive equilibrium for the induced $A$ market. Then $p^{(3)}$ is strictly positive.

Proof. Let $\left(\left(x^{i}\right)_{i \in N},\left(y^{i}\right)_{i \in N}, p\right)$ be a competitive equilibrium for the induced $A$-market. By the market clearing condition we have

$$
\sum_{i \in N} x^{i}=\sum_{i \in N} y^{i}+\left(0, e^{N}, 0, e^{N}, e^{N}\right)
$$

and by profit maximization $p \cdot y^{i}=0$ for all $i \in N$. By the definition of the production set for each $i \in N$ there exist $\gamma_{S}^{i 1}, \gamma_{S}^{i 2}, \gamma_{S}^{i 3} \geq 0$ for all $S \in \mathcal{N}, u_{S}^{i 1}, u_{S}^{i 2}, u_{S}^{i 3} \in C^{S}$ for all $S \in \mathcal{N} \backslash\{N\}$ and $\tilde{u}_{N}^{i 1}, \tilde{u}_{N}^{i 2}, \tilde{u}_{N}^{i 3} \in \tilde{C}^{N}$ such that

$$
\begin{aligned}
y^{i}= & \sum_{S \in \mathcal{N} \backslash\{N\}}\left(\sum_{j=1}^{3} \gamma_{S}^{i j} u_{S}^{i j},-\gamma_{S}^{i 1} e^{S}, \sum_{j=1}^{3} \gamma_{S}^{i j} u_{S}^{i j},-\left(\gamma_{S}^{i 1}+\gamma_{S}^{i 2}\right) e^{S},-\left(\gamma_{S}^{i 1}+\gamma_{S}^{i 3}\right) e^{S}\right) \\
& +\left(\sum_{j=1}^{3} \gamma_{N}^{i j} P_{A}\left(\tilde{u}_{N}^{i j}\right),-\gamma_{N}^{i 1} e^{N}, \sum_{j=1}^{3} \gamma_{N}^{i j} \tilde{u}_{N}^{i j},-\left(\gamma_{N}^{i 1}+\gamma_{N}^{i 2}\right) e^{N},-\left(\gamma_{N}^{i 1}+\gamma_{N}^{i 3}\right) e^{N}\right) .
\end{aligned}
$$

As $P_{A}\left(\tilde{C}^{N}\right)=C^{N}$ there exist $u_{N}^{i j} \in C^{N}$ such that $P_{A}\left(\tilde{u}_{N}^{i j}\right)=u_{N}^{i j}$ for $j=1,2,3$. Thus, we have for all $i \in N$

$$
\begin{aligned}
y^{i}= & \sum_{S \in \mathcal{N} \backslash\{N\}}\left(\sum_{j=1}^{3} \gamma_{S}^{i j} u_{S}^{i j},-\gamma_{S}^{i 1} e^{S}, \sum_{j=1}^{3} \gamma_{S}^{i j} u_{S}^{i j},-\left(\gamma_{S}^{i 1}+\gamma_{S}^{i 2}\right) e^{S},-\left(\gamma_{S}^{i 1}+\gamma_{S}^{i 3}\right) e^{S}\right) \\
& +\left(\sum_{j=1}^{3} \gamma_{N}^{i j} u_{N}^{i j},-\gamma_{N}^{i 1} e^{N}, \sum_{j=1}^{3} \gamma_{N}^{i j} \tilde{u}_{N}^{i j},-\left(\gamma_{N}^{i 1}+\gamma_{N}^{i 2}\right) e^{N},-\left(\gamma_{N}^{i 1}+\gamma_{N}^{i 3}\right) e^{N}\right) .
\end{aligned}
$$


By the definition of the consumption set we need to have $x^{(2) i}=x^{(4) i}=x^{(5) i}=0$ for all $i \in N$. Hence, for all $i \in N$, we obtain, using the market clearing condition and the definition of the production sets, for all coalitions $S \in \mathcal{N}$

$$
\begin{aligned}
\sum_{T \subseteq N} \gamma_{T}^{i 1} e^{T} & =e^{S}, \\
\sum_{T \subseteq N}\left(\gamma_{T}^{i 1}+\gamma_{T}^{i 2}\right) e^{T} & =e^{S}, \\
\sum_{T \subseteq N}\left(\gamma_{T}^{i 1}+\gamma_{T}^{i 3}\right) e^{T} & =e^{S} .
\end{aligned}
$$

It follows that $\gamma_{S}^{i 2}=\gamma_{S}^{i 3}=0$ for all $i \in N$ and for all $S \in \mathcal{N}$ and that for some $i \in N$ and some $S \in \mathcal{N}$ we have $\gamma_{S}^{i 1}>0$.

Suppose now, that $p_{i}^{(3)}=0$ for at least one $i \in N$. We show, that this leads to a contradiction.

First observe: If $p_{i}^{(3)}=0$ for one $i \in N$, then $p_{k}^{(3)}=0$ for all $k \in N$.

To see this suppose $p_{k}^{(3)}>0$ for some $k \in N$. For every individual $j \in N$ the consumption bundle $x^{j}$ maximizes his utility function over his budget set $\left\{\hat{x}^{j} \in\right.$ $\left.X^{j} \mid p \cdot \hat{x}^{j} \leq p \cdot \omega^{j}\right\}$. This implies, if $p_{i}^{(3)}=0$ that agent $j$ does not consume any good that has a positive price. If he did so, this would decrease his available budget whereas he can reach the same utility from consuming good $i$ that is for free. Precisely $p_{i}^{(3)}=0$ implies $x_{k}^{(3) j}=0$ for all $j \in N$ and for all $k \in N$ such that $k \neq i$ and $p_{k}^{(3)}>0$.

However, the market clearing condition and the definition of the production set require

$$
\sum_{j \in N} x^{(3) j}=\sum_{S \in \mathcal{N} \backslash\{N\}} \gamma_{S}^{i 1} u_{S}^{i 1}+\gamma_{N}^{i 1} \tilde{u}_{N}^{i 1} \gg 0,
$$

since $u_{S}^{i 1} \in C^{S} \subseteq \mathbb{R}_{++}^{S}$ and $\tilde{u}_{N}^{i 1} \geq u_{N}^{i 1} \in C^{N} \subseteq \mathbb{R}_{++}^{N}$. Hence, we obtain a contradiction and thus $p^{(3)}=0$.

Since $u^{j}\left(\check{x}^{j}\right)>u^{j}\left(\bar{x}^{j}\right)$ whenever $\check{x}_{j}^{(1) j}>\bar{x}_{j}^{(1) j}$ and $\check{x}^{(3) j}>\bar{x}^{(3) j}$, it follows from $p^{(3)}=0$ that $p_{j}^{(1)}$ must be positive. This holds for all $j \in N$, thus $p^{(1)} \gg 0$.

Since $C^{S} \subseteq \mathbb{R}_{++}^{S}$, it follows that $p^{(1)} \cdot u_{S}^{i 1}>0$. Since the maximal profits are equal to zero because of the convex-cone-technology, it must be true that

$$
p^{(1)} \cdot u_{S}^{i 1}-p^{(2)} \cdot e^{S}-p^{(4)} \cdot e^{S}-p^{(5)} \cdot e^{S}=0 .
$$


For any $j \in N$ choose $u \in C^{\{j\}} \cap \mathbb{R}_{++}^{\{j\}}$ and $\gamma>0$. Then

$$
\left(\gamma u, 0, \gamma u,-\gamma e^{\{j\}}, 0\right) \in Y^{j}
$$

and

$$
p \cdot\left(\gamma u, 0, \gamma u,-\gamma e^{\{j\}}, 0\right)=\gamma\left(p_{j}^{(1)} u-p_{j}^{(4)}\right) .
$$

Since $p^{(1)} \gg 0, p_{j}^{(4)}$ must be positive, because otherwise this would contradict the fact, that maximal profits are 0 . Thus, $p^{(4)} \gg 0$. Similarly $p^{(5)} \gg 0$. Therefore, from the equation $(\star)$ above we obtain using $-p^{(5)} \cdot e^{S}<0$ and $-p^{(2)} \cdot e^{S} \leq 0$

$$
p^{(1)} \cdot u_{S}^{i 1}-p^{(4)} \cdot e^{S}>0
$$

Hence, we have

$$
p \cdot\left(u_{S}^{i 1}, 0, u_{S}^{i 1},-e^{S}, 0\right)=p^{(1)} \cdot u_{S}^{i 1}+p^{(3)} \cdot u_{S}^{i 1}-p^{(4)} \cdot e^{S}=p^{(1)} \cdot u_{S}^{i 1}-p^{(4)} \cdot e^{S}>0 .
$$

But $\left(u_{S}^{i 1}, 0, u_{S}^{i 1},-e^{S}, 0\right) \in Y^{i}$ as it is of the form as points in the set $A_{S}^{2}$. This is a contradiction to the fact, that the maximal profits are zero. Thus $p^{(3)} \gg 0$.

We use this result to show the remaining Proposition that completes the proof of the theorem:

Proposition 4. Any payoff vector of a competitive equilibrium of the market $\mathcal{E}_{V, A, \varepsilon}$ is an element of the set $A$.

Proof. Suppose there exists a competitive equilibrium $\left(\left(x^{i}\right)_{i \in N}\left(y^{i}\right)_{i \in N}, p\right)$, such that $\left(u^{i}\left(x^{i}\right)\right)_{i \in N}=c^{N}$ with $c^{N} \notin A$.

From Lemma 1 we know that $c^{N}$ is in the inner core.

That Lemma 1 is applicable can be seen as follows: We know that $p \cdot \omega^{i}>0$. Otherwise agent $i$ would have a budget of 0 and we needed to have $p_{i}^{(2)}=p_{i}^{(4)}=$ $p_{i}^{(5)}=0$. This would mean that the production plan $\left(c^{\{i\}},-e^{\{i\}}, c^{\{i\}},-e^{\{i\}},-e^{\{i\}}\right)$ with $c^{\{i\}} \in C^{\{i\}}$ has strictly positive profits. This would be a contradiction. Thus, for all individuals $i \in N$ we have $p \cdot \omega^{i}>0$.

By Lemma 4 we know $p^{(3)} \gg 0$. Furthermore we know

$$
y=\sum_{i \in N} y^{i}=\left(P_{A}\left(\tilde{c}^{N}\right),-e^{N}, \tilde{c}^{N},-e^{N},-e^{N}\right)
$$

for some $\tilde{c}^{N} \in \tilde{C}^{N}$ satisfying $P_{A}\left(\tilde{c}^{N}\right)=c^{N}$ as any other production would contradict the market clearing condition in the $1^{\text {st }}$ group of $n$ coordinates. From the profit maximization 
we know that $\tilde{c}^{N}$ has to be chosen on the boundary of $\tilde{C}(N)$ and hence, since $c^{N} \notin A$, we have $\tilde{c}^{N} \gg c^{N}$. By the market clearing condition (for the $3^{r d}$ group of $n$ coordinates) we have

$$
\sum_{i \in N} x^{(3) i}=\tilde{c}^{N}
$$

Furthermore, by utility maximization we obtain

$$
c_{i}^{N}=x_{i}^{(3) i}+\varepsilon \sum_{j \neq i} x_{j}^{(3) i} .
$$

As $c^{N} \ll \tilde{c}^{N}$, equation $(\star \star \star)$ implies, that we have $x_{i}^{(3) i}<\tilde{c}_{i}^{N}$ for all $i \in N$.

Hence, for every $i \in N$ we have $\sum_{j \neq i} x_{i}^{(3) j}>0$. Thus, for every $i \in N$ there exists $j \neq i$ satisfying $x_{i}^{(3) j}>0$. Define a mapping $M: N \longrightarrow N$ in the following way: Every $i \in N$ is mapped to one $j \neq i$ satisfying $x_{i}^{(3) j}>0$. Then, we can find $k \in N$ and $t \in \mathbb{N}$ such that $M^{t}(k)=k$.

We use these results to show some constraints on the equilibrium prices: As $x_{k}^{(3) M(k)}>$ 0 , the utility maximization of agent $M(k)$ implies, that we have $p_{k}^{(3)} \leq \varepsilon p_{M(k)}^{(3)}$. Otherwise, agent $M(k)$ would not consume good $k$, but instead more of good $M(k)$. In the same way, we can show similar equations for other prices and obtain

$$
p_{k}^{(3)} \leq \varepsilon p_{M(k)}^{(3)} \leq \varepsilon^{2} p_{M^{2}(k)}^{(3)} \leq \ldots \leq \varepsilon^{t} p_{M^{t}(k)}^{(3)}=\varepsilon^{t} p_{k}^{(3)} .
$$

But $\varepsilon^{t}<1$. This is a contradiction.

As already mentioned before, assuming SPS is more restrictive than actually needed. Requiring the strict separation property for all points in the set $A$ can be weakened to requiring it only for the boundary points of the set $A$. In fact, we need for the construction of the auxiliary game $(N, \tilde{V})$ that outside the set $A$ the efficient boundary is strictly enlarged. This means the property that if we take $x \in V(N) \backslash A$, then $x$ being in the interior of $\tilde{V}(N)$ is the crucial property to eliminate equilibria with a payoff vector outside the set $A$. Using this weaker assumption allows a choice of the set $A$ as in Example 7. An example, where even this weaker version of the strict positive separability property is violated, and where our approach cannot be applied can be found in Figure 
5. Assume as before that we have always two players and that the coalitional function is given by $V(\{1\})=V(\{2\})=\{0\}-\mathbb{R}_{+}$and $V(\{1,2\})$ is given as indicated in Figure 5 .

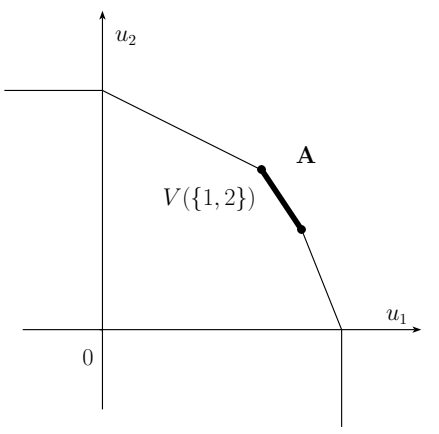

Example 7

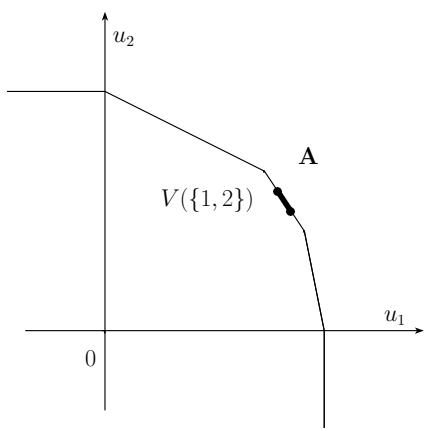

Example 9

Figure 5: Examples where SPS is not satisfied.

In contrast to Example 7, in Example 8 the set $A$ is chosen in such a way that it is a closed interval of a line segment connecting two neighboring corner points, but not the whole line segment. Because of the polyhedral structure none of the points in the set $A$ can be strictly separated from the set $V(\{1,2\})$ without the point.

Another important aspect of our result is the fact that the induced $A$-market is not determined uniquely. We have some freedom in different aspects of our construction and obtain a whole class of markets, that can be used to prove our main theorem:

- First, to define the induced $A$-market we use the auxiliary NTU game $(N, \tilde{V})$ where we enlarge the given NTU game $(N, V)$. For this enlargement we use for every inner core point one of its normal vectors. This normal vector is not always unique.

- Second, for the auxiliary game $(N, \tilde{V})$ we define the mapping $P_{A}$ which can be chosen in different ways. The important property is that for the points outside the given subset of the inner core, $A$, we have $P_{A}(z) \gg z$ for all $z \in I C(A) \backslash A$. Moreover, for points in the given set $A$ we require $P_{A}(z)=z$ for all $z \in A$.

- Third, we add to the utility function of the induced $A$-market an $\varepsilon$-term, that needs to be between certain bounds and hence is not determined uniquely. Moreover, we can choose different $\varepsilon$ for different players.

\section{Concluding Remarks}

In this paper we have continued the work of Shapley and Shubik (1975) and Qin (1993) to investigate competitive payoff vectors of markets that represent a cooperative game 
and their relation to solution concepts for cooperative games.

We extend the results of Qin (1993) to a large class of closed subsets of the inner core: Given an NTU market game we construct the induced $A$-market depending on a given closed subset of its inner core. This market represents the game and further has the given set as the set of payoff vectors of competitive equilibria. More precisely, inspired by the construction of the induced market of Billera and Bixby (1974) and by the markets that Qin (1993) uses to prove his two main results, we define a market in an appropriate way to generalize the results of Qin (1993) to a large class of closed subsets of the inner core. It turns out that this market is not determined uniquely and thus we obtain a whole class of markets that has the given closed subset of the inner core as the set of payoff vectors of competitive equilibria.

In the literature it was already known that one game can be represented by several markets, see Billera and Bixby (1974) or Qin (1993). Our work confirms that going from NTU games to markets some structural information is added that is not present in the NTU game. To a given NTU market game we can associate a huge class of markets that represents the NTU game. In particular, by choosing the structure, that we add, we can control the set of payoffs of competitive equilibria.

Another point of view on our results is to analyze situations where we start with given markets and consider the induced games. Looking at competitive equilibria and how they appear in the game, we observe that almost everything is possible. Depending on the specific market the set of competitive equilibrium payoff vectors might fill up the whole inner core or be almost any closed subset, in particular any single point. Hence, our result demonstrates that we can not expect to observe more game theoretic properties of competitive equilibria than knowing that competitive payoffs are in the inner core. Only by imposing additional structural assumptions on the markets, for example restricting the class of utility functions, we may observe additional game theoretic properties.

We establish a link between closed subsets of the inner core and competitive payoffs of certain economies. Extending the results of Qin (1993) to closed subsets of the inner core means in particular to establish a link for all solution concepts selecting closed subsets of the inner core. Therefore, our results can be seen as a market foundation of game theoretic solution concepts that select closed subsets of the inner core. For the particular class of bargaining games a more precise presentation of the idea of a market foundation can be found in Trockel $(1996,2005)$ and Brangewitz and Gamp (2011).

The result presented here includes the result of Qin (1993) for a single point in the inner core. This holds also in a very general setup by using monotone transformations of utilities in the same way as it was done in Qin (1993). Nevertheless, if we consider closed subsets of the inner core that contain more than a single point, the idea to transform the utilities seems not to work. Due to this fact we assume some separation properties on 
the game and the given closed subset of its inner core.

Furthermore, by investigating the NTU case we realized that a simple generalization of the approach of Shapley and Shubik (1975) in the framework of Qin (1993) does not work and we need to stay closer to the results on NTU games. More precisely, changing the utility function in the market, that Qin (1993) uses to prove his second result, in analogy to the TU case of Shapley and Shubik (1975) to

$$
u^{i}\left(x^{i}\right)=\min \left\{x_{i}^{(1) i}, \min _{u^{*} \in A}\left\{\frac{\left(\lambda^{u^{*}} \circ u^{*}\right) \cdot x^{(5) i}}{\lambda_{i}^{u^{*}}}\right\}\right\}
$$

does not lead to markets with the desired properties.

Having our result in mind there remains the open question if we can further weaken our assumptions such that the results can be proved for more general cases. Another interesting related line of research is to continue to look at the class of games that are linked to coalition production economies as analyzed by Inoue (2010b). Given a balanced NTU game Inoue (2010b) defines a coalition production economy such that this economy represents the game and has moreover the whole inner core as the set of competitive equilibrium payoff vectors. It remains an open question if one can find analogously to Qin (1993) and to this work a coalition production economy such that one inner core point or a certain subset of the inner core are competitive equilibrium payoff vectors in this coalition production economy. Moreover, it is interesting to compare the set of competitive equilibrium allocations of different market representations of a given NTU market game. Does there exist a general and more simple method to obtain desired competitive payoffs? Can we characterize a class of NTU games where this is possible? What happens if we restrict our attention for example to bargaining games? 


\section{References}

Arrow, K. J. and Debreu, G. (1954). Existence of an equilibrium for a competitive economy. Econometrica, 22(3):265-290.

Billera, L. J. (1974). On games without side payments arising from a general class of markets. Journal of Mathematical Economics, 1(2):129-139.

Billera, L. J. and Bixby, R. E. (1973). A characterization of polyhedreal market games. International Journal of Game Theory, 2:253-261.

Billera, L. J. and Bixby, R. E. (1974). Market representations of $n$-person games. Bulletin of the American Mathematical Society, 80(3):522-526.

Bonnisseau, J.-M. and Iehlé, V. (2007). Payoff-dependent balancedness and cores. Games and Economic Behavior, 61(1):1 - 26.

Brangewitz, S. and Gamp, J.-P. (2011). Inner core, asymmetric Nash bargaining solution and competitive payoffs. Unpublished.

de Clippel, G. and Minelli, E. (2005). Two remarks on the inner core. Games and Economic Behavior, 50(2):143 - 154.

Iehlé, V. (2004). Transfer rate rules and core selections in NTU games. Economics Bulletin, 3(42):1 - 10.

Inoue, T. (2010a). Coincidence theorem and the nonemptiness of the inner core. Unpublished.

Inoue, T. (2010b). Representation of NTU games by coalition production economies. Unpublished.

Qin, C.-Z. (1993). A conjecture of Shapley and Shubik on competitive outcomes in the cores of NTU market games. International Journal of Game Theory, 22:335-344.

Qin, C.-Z. (1994). The inner core of an $n$-person game. Games and Economic Behavior, $6(3): 431-444$.

Qin, C.-Z. and Shubik, M. (2009). Selecting a unique competitive equilibrium with default penalties. Cowles Foundation Discussion Papers 1712, Cowles Foundation for Research in Economics, Yale University.

Shapley, L. S. (1987). Mathematics 147, game theory: Notes. UCLA, Dept. of Mathematics. 
Shapley, L. S. and Shubik, M. (1969). On market games. Journal of Economic Theory, $1: 9-25$.

Shapley, L. S. and Shubik, M. (1975). Competitive outcomes in the cores of market games. International Journal of Game Theory, 4(4):229-237.

Shubik, M. (1985). Game theory and the social sciences concepts and solutions. MIT Press.

Sun, N., Trockel, W., and Yang, Z. (2008). Competitive outcomes and endogenous coalition formation in an n-person game. Journal of Mathematical Economics, 44(78):853-860.

Trockel, W. (1996). A Walrasian approach to bargaining games. Economics Letters, 51(3):295-301.

Trockel, W. (2005). Core-equivalence for the Nash bargaining solution. Economic Theory, 25(1):255-263. 


\section{A Appendix}

\section{A.1 Proof of Lemma 1}

For the proof of Lemma 1 we follow the idea of de Clippel and Minelli (2005).

Proof. Let $\left(\hat{x}^{i}\right)_{i \in N}$ and $\left(\hat{y}^{i}\right)_{i \in N}$ be a competitive equilibrium allocation at a price $\hat{p} \in$ $\mathbb{R}_{+}^{\ell} \backslash\{0\}$. For each individual $i \in N$ define the set

$$
C^{i}=\left\{(u, m) \in \mathbb{R}^{2} \mid \exists z^{i} \in X^{i}: u \leq u^{i}\left(z^{i}\right)-u^{i}\left(\hat{x}^{i}\right), m \leq \hat{p} \cdot\left(\omega^{i}+\hat{y}^{i}-z^{i}\right)\right\}
$$

By the concavity of $u^{i}$, this set is convex. On the other hand, $C^{i} \cap \mathbb{R}_{++}^{2}=\emptyset$, as $\hat{x}^{i}$ is optimal for individual $i$ in his budget set.

Suppose $(u, m) \in C^{i}$ and $(u, m) \gg 0$, then there exists $z^{i} \in X^{i}$ with $\left.u\left(\hat{x}^{i}\right)<u^{(} z^{i}\right)$ and $\hat{p} \cdot z^{i}<\hat{p} \cdot\left(\omega^{i}+\hat{y}^{i}\right)$ which means $z^{i}$ gives individual $i$ a higher utility as $\hat{x}^{i}$ and is affordable under the price system $\hat{p}$. This is in contradiction to the optimality of $\hat{x}^{i}$.

By the separating hyperplane theorem there exists a non-zero, non-negative vector $\left(\alpha^{i}, \beta^{i}\right) \in$ $\mathbb{R}_{+}^{2}$ such that we can separate 0 from $C^{i}$ and obtain

$$
\alpha^{i} u^{i}\left(\hat{x}^{i}\right) \geq \alpha^{i} u^{i}\left(z^{i}\right)-\beta^{i} \hat{p} \cdot\left(z^{i}-\omega^{i}-\hat{y}^{i}\right)
$$

for all $z^{i} \in X^{i}$.

As $\hat{p} \cdot \omega^{i}>0$, it follows from the above inequality that we have $\alpha^{i}>0$.

To see this suppose $\alpha^{i}=0\left(\beta^{i}>0\right)$. Then, as in equilibrium $\hat{p} \cdot \hat{y}^{i}=0$, we obtain from the above inequality

$$
0 \leq \hat{p} \cdot\left(z^{i}-\omega^{i}-\hat{y}^{i}\right) \quad \text { for all } z^{i} \in X^{i}
$$

which is not true, as $0 \in X^{i}$ and $\hat{p} \cdot \hat{y}^{i}=0$. Thus $\alpha^{i}>0$.

We can assume $\alpha^{i}=1$ without the loss of generality. Moreover, monotonicity and locally non-satiation of the utility function imply that $\beta^{i}>0$. Let $\lambda^{i}=\frac{1}{\beta^{i}}$. Summing up over all $i \in S$ we obtain

$$
\sum_{i \in S} \lambda^{i} u^{i}\left(\hat{x}^{i}\right) \geq \sum_{i \in S} \lambda^{i} u^{i}\left(z^{i}\right)-\hat{p} \cdot \sum_{i \in S}\left(z^{i}-\omega^{i}-\hat{y}^{i}\right)
$$

for all $S \subseteq N$ and for all $z^{i} \in \mathbb{R}_{+}^{\ell}$ with $i \in S$. 
If a coalition $S$ could $\lambda$-improve on $x$ with $\left(\bar{x}^{i}\right)_{i \in S}$ (with the production plan $\left.\bar{y}^{i} \in Y^{i}\right)$, then the previous inequality would be violated, because we have, due to feasibility,

$$
\sum_{i \in S}\left(\bar{x}^{i}-\omega^{i}-\bar{y}^{i}\right) \leq 0
$$

and thus we obtain a contradiction by

$$
\begin{aligned}
\sum_{i \in S} \lambda^{i} u^{i}\left(\bar{x}^{i}\right) & >\sum_{i \in S} \lambda^{i} u^{i}\left(\hat{x}^{i}\right) \\
& \geq \sum_{i \in S} \lambda^{i} u^{i}\left(\bar{x}^{i}\right)-\hat{p} \cdot \sum_{i \in S}\left(\bar{x}^{i}-\omega^{i}-\hat{y}^{i}\right) \\
& \geq \sum_{i \in S} \lambda^{i} u^{i}\left(\bar{x}^{i}\right)-\hat{p} \cdot \sum_{i \in S}\left(\bar{x}^{i}-\omega^{i}\right) \\
& \geq \sum_{i \in S} \lambda^{i} u^{i}\left(\bar{x}^{i}\right)-\hat{p} \cdot \sum_{i \in S} \bar{y}^{i} \\
& \geq \sum_{i \in S} \lambda^{i} u^{i}\left(\bar{x}^{i}\right) .
\end{aligned}
$$




\section{A.2 Proof of Claim 1}

Proof. We show

$$
x_{i}^{(1) i}=x_{i}^{(3) i}
$$

by contradiction. Then it immediately follows from $u^{i}\left(x^{i}\right)=c_{i}^{N}$ that

$$
x_{i}^{(1) i}=x_{i}^{(3) i}=c_{i}^{N} \text {. }
$$

Suppose $x_{i}^{(3) i}>x_{i}^{(1) i}$. This cannot be utility maximizing in the presence of strictly positive prices. If player $i$ consumes a little bit less of the $i^{\text {th }}$ good of the $3^{\text {rd }}$ group of $n$ goods and invests the - not anymore used - additional budget in the $i^{\text {th }}$ good of the $1^{\text {st }}$ group of $n$ goods, then he can strictly increase his utility.

Precisely, from the assumption $u^{i}\left(x^{i}\right)=c_{i}^{N}$ and $x_{i}^{(3) i}>x_{i}^{(1) i}$ it follows that $x_{i}^{(1) i}=c_{i}^{N}$. For $\delta$ sufficiently small, i.e. $0<\delta<x_{i}^{(3) i}-x_{i}^{(1) i}$, player $i$ can increase his utility by consuming $\delta$ less of the $i^{\text {th }}$ good of the $3^{r d}$ group of $n$ goods and increasing the consumption in the $i^{\text {th }}$ good of the $1^{\text {st }}$ group of $n$ goods by $\frac{p_{i}^{(3)}}{p_{i}^{(1)}} \delta$. To consume $\left(x^{(1) i}+\frac{p_{i}^{(3)}}{p_{i}^{(1)}} \delta e^{\{i\}}, 0, x^{(3) i}-\delta e^{\{i\}}, 0\right)$ is still budget feasible for player $i$, because

$$
p^{(1)}\left(x^{(1) i}+\frac{p_{i}^{(3)}}{p_{i}^{(1)}} \delta e^{\{i\}}\right)+p^{(3)}\left(x^{(3) i}-\delta e^{\{i\}}\right)=p^{(1)} x^{(1) i}+p^{(3)} x^{(3) i} \leq p \cdot \omega^{i}
$$

Hereby, the last inequality follows from the budget feasibility of $x^{i}$. Moreover, the utility of consumer $i$ strictly increases, since

$$
u^{i}\left(x^{(1) i}+\frac{p_{i}^{(3)}}{p_{i}^{(1)}} \delta, 0, x^{(3) i}-\delta, 0\right)>x_{i}^{(1) i}=u^{i}\left(x^{(1) i}, 0, x^{(3) i}, 0\right)
$$

by the choice of $\delta$. This is a contradiction to the assumption that $x^{i}$ is utility maximizing. Hence, we have $x_{i}^{(3) i} \leq x_{i}^{(1) i}$.

By exchanging the roles of $x_{i}^{(1) i}$ and $x_{i}^{(3) i}$ we can analogously show $x_{i}^{(3) i} \geq x_{i}^{(1) i}$. Therefore, we have $x_{i}^{(3) i}=x_{i}^{(1) i}$. 